\title{
Role of thermal field in entanglement harvesting between two accelerated Unruh-DeWitt detectors
}

\author{
Dipankar Barman, Subhajit Barman and Bibhas Ranjan Majhi \\ Department of Physics, Indian Institute of Technology Guwahati, \\ Guwahati 781039, Assam, India \\ E-mail: dipankar1998@iitg.ac.in, subhajit.b@iitg.ac.in, \\ bibhas.majhi@iitg.ac.in
}

ABSTRACT: We investigate the effects of field temperature $T^{(f)}$ on the entanglement harvesting between two uniformly accelerated detectors. For their parallel motion, the thermal nature of fields does not produce any entanglement, and therefore, the outcome is the same as the non-thermal situation. On the contrary, $T^{(f)}$ affects entanglement harvesting when the detectors are in anti-parallel motion, i.e., when detectors $A$ and $B$ are in the right and left Rindler wedges, respectively. While for $T^{(f)}=0$ entanglement harvesting is possible for all values of $A$ 's acceleration $a_{A}$, in the presence of temperature, it is possible only within a narrow range of $a_{A}$. In $(1+1)$ dimensions, the range starts from specific values and extends to infinity, and as we increase $T^{(f)}$, the minimum required value of $a_{A}$ for entanglement harvesting increases. Moreover, above a critical value $a_{A}=a_{c}$ harvesting increases as we increase $T^{(f)}$, which is just opposite to the accelerations below it. There are several critical values in $(1+3)$ dimensions when they are in different accelerations. Contrary to the single range in $(1+1)$ dimensions, here harvesting is possible within several discrete ranges of $a_{A}$. Interestingly, for equal accelerations, one has a single critical point, with nature quite similar to $(1+1)$ dimensional results. We also discuss the dependence of mutual information among these detectors on $a_{A}$ and $T^{(f)}$.

Keywords: Classical Theories of Gravity, Thermal Field Theory

ArXiv EPrint: 2104.11269 


\section{Contents}

1 Introduction 1

2 Model set-up: a summary of the main results 4

3 Accelerated observers in a thermal bath $\quad 7$

3.1 Coordinate systems 7

3.2 Scalar field decomposition corresponding to an accelerated observer 8

$3.2 .1 \quad(1+1)$ dimensions 9

$\begin{array}{lll}3.2 .2 & (1+3) \text { dimensions } & 10\end{array}$

$\begin{array}{lll}3.3 & \text { Two-point correlators for thermal field } & 11\end{array}$

$\begin{array}{lll}3.3 .1 & (1+1) \text { dimensions } & 11\end{array}$

$\begin{array}{lll}3.3 .2 & (1+3) \text { dimensions } & 12\end{array}$

4 Entanglement harvesting $\quad \mathbf{1 3}$

$\begin{array}{ll}4.1 \text { Parallel acceleration: no harvesting } & 15\end{array}$

$\begin{array}{lll}4.1 .1 & (1+1) \text { dimensions } & 15\end{array}$

$\begin{array}{lll}4.1 .2 & (1+3) \text { dimensions } & 16\end{array}$

$\begin{array}{lll}4.2 & \text { Anti-parallel accelerations: harvesting possible } & 17\end{array}$

$\begin{array}{lll}4.2 .1 & (1+1) \text { dimensions } & 17\end{array}$

$\begin{array}{lll}4.2 .2 & (1+3) \text { dimensions } & 24\end{array}$

5 Mutual information $\quad 29$

$\begin{array}{llr}5.1 & \text { Parallel acceleration } & 29\end{array}$

$\begin{array}{lll}5.1 .1 & (1+1) \text { dimensions } & 29\end{array}$

$\begin{array}{lll}5.1 .2 & (1+3) \text { dimensions } & 30\end{array}$

5.2 Anti-parallel acceleration 31

$\begin{array}{lll}5.2 .1 & (1+1) \text { dimensions } & 31\end{array}$

$\begin{array}{lll}5.2 .2 & (1+3) \text { dimensions } & 32\end{array}$

6 Discussion $\quad 32$

\section{Introduction}

Quantum entanglement is a fascinating phenomenon distinguishing quantum and classical physics and has acquired immense practical importance through quantum communication and cryptography $[1,2]$. There has been a growing interest to realize entanglement and understand its nature for relativistic particles in flat and in curved spacetimes, see [3-16]. In this regard, entanglement extraction from the quantum field vacuum became very important for the fundamental understandings of the vacuum and the background spacetime. 
This phenomenon is better known as entanglement harvesting [4, 9, 17-21], which states that from quantum fields, one can harvest entanglement among atoms or other suitable systems interacting with the field. Entanglement harvesting acquires additional significance from the possibility of its experimental verification and utilization of the extracted entanglement in quantum information-related purposes [22-24]. In the pioneering works by Reznik $[4,18]$, he provided an understanding of entanglement harvesting in a system of two accelerated atoms by considering them as point-like two-level Unruh-DeWitt particle detectors [25] interacting with background massless scalar field. Unruh-DeWitt particle detectors are hypothetical detectors, conceptualized to understand the Unruh effect [26, 27]. He showed that entanglement extraction was possible between two causally disconnected anti-parallelly accelerated detectors in the separate Rindler wedges, signifying the quantum vacuum's role for harvested entanglement.

There has been plenty of works related to entanglement harvesting in different spacetime backgrounds, and one can look into [10, 28-37] for a thorough anthology. In all these works, one usually investigates a system composed of two initially non-entangled detectors interacting with the background quantum field. The aim is to study the later time density matrix only for the detectors, where the field degrees of freedom are being traced out. For entanglement harvesting, i.e., for the two qubits to be entangled, it is necessary to have negative eigenvalues of the partial transposition of the detector density matrix. It should be noted that in initial works $[4,9]$ the authors found these eigenvalues to be dependent on quantities estimated from positive frequency Wightman functions connecting different spacetime events of the same or different detectors. However, recent rigorous investigations [38-40] have suggested proper time ordering into the picture, which results in the inception of Feynman propagator rather than Wightman function in the estimation of the eigenvalues. These recent methods provide a meticulous and more general formulation for the understanding of entanglement harvesting. However, even with these changes, most previous perceptions regarding entanglement extraction corresponding to accelerated observers - like one can harvest entanglement between two anti-parallelly accelerated detectors but not for parallelly accelerated observers - remain the same. Although, the individual contributions of the retarded Green's function and the Wightman function from the Feynman propagator remain an interesting arena to venture further.

On the other hand, the effects of a thermal bath on entanglement harvesting remain equally interesting (see [41, 42]). In nature, an environment with thermal background is much more practical. Including the thermal nature in the model and investigating the effects in the physical quantities will approach a more realistic situation and thereby help to know the exact features of our surroundings. In this regard, one may mention that the thermal nature of fields has already been included in various investigations related to Unruh-De Witt detectors; like calculation of response functions in case of a single detector, [43-46], and two entangled detectors [47]. In [41, 42, 48] it is predicted that the entanglement extraction gets depleted with increasing temperature of the thermal field. Then it will be pretty fascinating to study the situation of entanglement harvesting for accelerated observers interacting with thermal fields, which is not there in the literature up 
to our knowledge. In this regard, in literature $[38,40]$ the Feynman propagators and the positive frequency Wightman functions, necessary to understand entanglement extraction, are estimated in the Minkowski position space. Then for the calculations relating to accelerated observers, the relevant transformations to Rindler coordinates are made to those Green's functions, and this method does not encounter any particular issue. However, consider a similar description of the Feynman propagators and the Wightman functions for thermal fields. The resulting Green's functions do not remain time translational invariant with the detectors' proper times. In [47] the authors have discussed this issue and considered Rindler modes with the vacuum for the Unruh modes to describe the Wightman functions corresponding to accelerated observers in a thermal bath, which are time translational invariant. This method, in line with the chain of thoughts also presented in [39], circumvents the previously mentioned issue by expressing the Green's functions in terms of modes and their momentum space integrals rather than a position space representation.

In this work, we are going to investigate the condition for entanglement harvesting and study the concurrence $[38,40,49]$, a measure of the harvested entanglement, for two accelerated Unruh-DeWitt detectors interacting with a massless thermal scalar field in $(1+1)$ and $(1+3)$ dimensions. In particular, we consider the interaction between the two-level point-like detectors and the scalar field to be of monopole type. We observe that the specific form of this monopole moment operator is not needed to understand the role of the spacetime trajectories and the thermal bath in entanglement extraction. We use the prescription as provided in [47] for the construction of the Green's functions and follow the formulation of articles $[38,40]$ for entanglement harvesting. We arrive at the same assertions that entanglement extraction is possible only for the anti-parallelly accelerated detectors and not for the parallelly accelerated ones, and also encounter the phenomena of degrading entanglement extraction with increasing temperature of the thermal bath [41, 42]. However, the situation is a bit more involved in our case as we observe this degradation happening in the low acceleration regimes. We observe that in $(1+1)$ dimensions, above a specific value of acceleration, thermal bath enhances the entanglement harvesting. While below this specific acceleration, the same is degraded with increasing background field temperature. Therefore, for anti-parallel detectors, a notion of phase transition-like phenomena is encountered around a critical acceleration value. However, we found that the range of acceleration in which entanglement harvesting is possible is consistently decreasing with the increasing temperature of the thermal bath. In $(1+3)$ dimensions, for equal accelerations of the detectors, the characteristics of concurrence are the same as the $(1+1)$ dimensional case. However, for unequal detectors' accelerations, we encounter multiple transition points for $a_{A}$, the acceleration of detector $A$, when the acceleration $a_{B}$ of detector $B$ is fixed. We notice that between these transition points, the nature of the concurrence flips with the temperature of the thermal bath compared to the adjacent regions. In that case, contrary to the single range of $a_{A}$ in $(1+1)$ dimensions, we now have discrete ranges of acceleration $a_{A}$ for entanglement harvesting to be possible for a fixed temperature of the thermal bath. It is observed that a non-vanishing contribution is coming from the retarded part of the Feynman propagator when the detectors have unequal magnitudes of accelerations. We 
have also investigated the nature of mutual information $\mathcal{M}$ among the two detectors. Here $\mathcal{M}$ is non-vanishing for parallel motion, whereas it vanishes in the anti-parallel situation. For a non-vanishing case, $\mathcal{M}$ increases with the increase in temperature of the background field. On the other hand, it decreases with the growth of acceleration of the first detector.

In section 2 we begin with a brief discussion of our model set-up of two two-level pointlike atomic detectors interacting with the vacuum massless scalar field through monopole couplings. We consider the detectors initially in their separable ground state. This section also discusses the entanglement harvesting condition and entanglement measures obtained from the final form of the detector density matrix. In section 3 we elucidate on accelerated observers in a thermal bath and provide the expressions of the Green's functions for the situation of parallelly and anti-parallelly accelerating observers, considering the Rindler field decomposition with the Unruh operators and Unruh mode vacuum. Subsequently, in section 4 the condition for entanglement harvesting is analyzed first for two parallelly and then for two anti-parallelly accelerated observers in a thermal bath using the Green's functions of section 3. In this section, we study the entanglement measure concurrence and, in section 5 investigate the mutual information between the two detectors to discuss the notable outcomes. We conclude this article in section 6 with a discussion of our results.

\section{Model set-up: a summary of the main results}

Having said our motivation in the introduction, let us now talk about the model which will be dealt with in this article. The model on which we will concentrate here was originally introduced in [38-40]. Therefore, without going into the details of this and the derivation of the required formulas, the final expressions which are needed in this paper will be summarised here. Also a brief idea of the model will be given in order to be acquainted with the notations and symbols, we will use.

We consider two two-level point-like Unruh-DeWitt detectors, one carried by Alice and denoted by $A$. Another denoted by $B$, which is carried by Bob. The detector states are denoted by $\left|E_{n}^{j}\right\rangle$, with the symbols denoting the $n^{\text {th }}$ state of $j^{\text {th }}$ detector, i.e., $j=A, B$ and $n=0,1$. These states are non degenerate so that $E_{1}^{j} \neq E_{0}^{j}$, and it is assumed that $\Delta E^{j}=E_{1}^{j}-E_{0}^{j}>0$. We consider these detectors to be interacting through monopole interactions $m_{j}\left(\tau_{j}\right)$ with a massless, minimally coupled scalar field $\Phi(X)$. The interaction action corresponding to this system is

$$
S_{\mathrm{int}}=\int_{-\infty}^{\infty}\left[c_{A} \kappa_{A}\left(\tau_{A}\right) m_{A}\left(\tau_{A}\right) \Phi\left(X_{A}\left(\tau_{A}\right)\right) d \tau_{A}+c_{B} \kappa_{B}\left(\tau_{B}\right) m_{B}\left(\tau_{B}\right) \Phi\left(X_{B}\left(\tau_{B}\right)\right) d \tau_{B}\right],
$$

where, $c_{j}$ denote the couplings between the individual detectors and the scalar field, $\kappa_{j}\left(\tau_{j}\right)$ the switching functions and $\tau_{j}$ the individual detector proper times. The initial detector field state is considered to be the one at the asymptotic past, denoted by $|i n\rangle=|0\rangle\left|E_{0}^{A}\right\rangle\left|E_{0}^{B}\right\rangle$. Whereas the final detector state at asymptotic future is $|o u t\rangle=$ $T\left\{e^{i S_{\text {int }}}|i n\rangle\right\}$. Treating the coupling constants $c_{j}$ perturbatively and tracing out the field degrees of freedoms one can obtain the density matrix corresponding to the final state in 
the basis of $\left\{\left|E_{1}^{A}\right\rangle\left|E_{1}^{B}\right\rangle,\left|E_{1}^{A}\right\rangle\left|E_{0}^{B}\right\rangle,\left|E_{0}^{A}\right\rangle\left|E_{1}^{B}\right\rangle,\left|E_{0}^{A}\right\rangle\left|E_{0}^{B}\right\rangle\right\}$ as

$$
\rho_{A B}=\left[\begin{array}{cccc}
0 & 0 & 0 & c_{A} c_{B} \varepsilon \\
0 & c_{A}^{2} P_{A} & c_{A} c_{B} P_{A B} & c_{A}^{2} W_{A}^{(N)}+c_{A} c_{B} W_{A}^{(S)} \\
0 & c_{A} c_{B} P_{A B}^{*} & c_{B}^{2} P_{B} & c_{B}^{2} W_{B}^{(N)}+c_{A} c_{B} W_{B}^{(S)} \\
c_{A} c_{B} \varepsilon^{*} & c_{A}^{2} W_{A}^{(N) *}+c_{A} c_{B} W_{A}^{(S) *} & c_{B}^{2} W_{B}^{(N) *}+c_{A} c_{B} W_{B}^{(S) *} & 1-\left(c_{A}^{2} P_{A}+c_{B}^{2} P_{B}\right)
\end{array}\right]+\mathcal{O}\left(c^{4}\right),
$$

where, the expressions of $P_{j}, \varepsilon, P_{A B}, W_{j}^{(N)}$, and $W_{j}^{(S)}$ are given by

$$
\begin{aligned}
P_{j} & =\left|\left\langle E_{1}^{j}\left|m_{j}(0)\right| E_{0}^{j}\right\rangle\right|^{2} \mathcal{I}_{j} \\
\varepsilon & =\left\langle E_{1}^{B}\left|m_{B}(0)\right| E_{0}^{B}\right\rangle\left\langle E_{1}^{A}\left|m_{A}(0)\right| E_{0}^{A}\right\rangle \mathcal{I}_{\varepsilon} \\
P_{A B} & =\left\langle E_{1}^{A}\left|m_{A}(0)\right| E_{0}^{A}\right\rangle\left\langle E_{1}^{B}\left|m_{B}(0)\right| E_{0}^{B}\right\rangle^{\dagger} \mathcal{I}_{A B} \\
W_{j}^{(N)} & =\left\langle E_{1}^{j}\left|m_{j}(0)\right| E_{0}^{j}\right\rangle\left[\left(\left\langle E_{1}^{j}\left|m_{j}(0)\right| E_{1}^{j}\right\rangle-\left\langle E_{0}^{j}\left|m_{j}(0)\right| E_{0}^{j}\right\rangle\right) \mathcal{I}_{j, 1}^{(N)}-i\left\langle E_{0}^{j}\left|m_{j}(0)\right| E_{0}^{j}\right\rangle \mathcal{I}_{j, 2}^{(N)}\right] \\
W_{j}^{(S)} & =-i\left\langle E_{1}^{j}\left|m_{j}(0)\right| E_{0}^{j}\right\rangle\left\langle E_{0}^{j^{\prime}}\left|m_{j^{\prime}}(0)\right| E_{0}^{j^{\prime}}\right\rangle \mathcal{I}_{j}^{(S)},
\end{aligned}
$$

where $j^{\prime} \neq j$ and the quantities $\mathcal{I}$,s are given by

$$
\begin{aligned}
\mathcal{I}_{j} & =\int_{-\infty}^{\infty} d \tau_{j}^{\prime} \int_{-\infty}^{\infty} d \tau_{j} e^{-i \Delta E^{j}\left(\tau_{j}^{\prime}-\tau_{j}\right)} G_{W}\left(X_{j}^{\prime}, X_{j}\right), \\
\mathcal{I}_{\varepsilon} & =-i \int_{-\infty}^{\infty} d \tau_{B}^{\prime} \int_{-\infty}^{\infty} d \tau_{A} e^{i\left(\Delta E^{B} \tau_{B}^{\prime}+\Delta E^{A} \tau_{A}\right)} G_{F}\left(X_{B}^{\prime}, X_{A}\right), \\
\mathcal{I}_{A B} & =\int_{-\infty}^{\infty} d \tau_{B}^{\prime} \int_{-\infty}^{\infty} d \tau_{A} e^{i\left(\Delta E^{A} \tau_{A}-\Delta E^{B} \tau_{B}^{\prime}\right)} G_{W}\left(X_{B}^{\prime}, X_{A}\right), \\
\mathcal{I}_{j, 1}^{(N)} & =\int_{-\infty}^{\infty} d \tau_{j}^{\prime} \int_{-\infty}^{\infty} d \tau_{j} e^{i \Delta E^{j} \tau_{j}} \theta\left(\tau_{j}^{\prime}-\tau_{j}\right) G_{W}\left(X_{j}^{\prime}, X_{j}\right), \\
\mathcal{I}_{j, 2}^{(N)} & =\int_{-\infty}^{\infty} d \tau_{j}^{\prime} \int_{-\infty}^{\infty} d \tau_{j} e^{i \Delta E^{j} \tau_{j}} G_{R}\left(X_{j}, X_{j}^{\prime}\right), \\
\mathcal{I}_{j}^{(S)} & =\int_{-\infty}^{\infty} d \tau_{j^{\prime}}^{\prime} \int_{-\infty}^{\infty} d \tau_{j} e^{i \Delta E^{j} \tau_{j}} G_{R}\left(X_{j}, X_{j^{\prime}}^{\prime}\right)
\end{aligned}
$$

Here in these expressions the switching functions have not appeared as we have considered them $\kappa_{j}\left(\tau_{j}\right)=1$; i.e. the detectors are interacting with fields all the time. On the other hand, the quantities $G_{W}\left(X_{j}, X_{j^{\prime}}\right), G_{F}\left(X_{j}, X_{j^{\prime}}\right)$, and $G_{R}\left(X_{j}, X_{j^{\prime}}\right)$ respectively denote the positive frequency Wightman function with $X_{j}>X_{j^{\prime}}$, the Feynman propagator, and the retarded Green's function, and their expressions are

$$
\begin{aligned}
G_{W}\left(X_{j}, X_{j^{\prime}}\right) & \equiv\left\langle 0_{M}\left|\Phi\left(X_{j}\right) \Phi\left(X_{j^{\prime}}\right)\right| 0_{M}\right\rangle, \\
G_{F}\left(X_{j}, X_{j^{\prime}}\right) & \equiv-i\left\langle 0_{M}\left|T\left\{\Phi\left(X_{j}\right) \Phi\left(X_{j^{\prime}}\right)\right\}\right| 0_{M}\right\rangle, \\
G_{R}\left(X_{j}, X_{j^{\prime}}\right) & \equiv i \theta\left(t-t^{\prime}\right)\left\langle 0_{M}\left|\left[\Phi\left(X_{j^{\prime}}\right), \Phi\left(X_{j}\right)\right]\right| 0_{M}\right\rangle .
\end{aligned}
$$

The details of the derivation can be followed from [38]. The condition for entanglement, based on a general analysis for a bipartite system [50,51], is obtained from the negative eigenvalue of the partial transposition of the reduced density matrix from eq. (2.2), and this condition results in

$$
P_{A} P_{B}<|\varepsilon|^{2},
$$


which can also be cast, in terms of the integrals, into the form [38, 40]

$$
\mathcal{I}_{A} \mathcal{I}_{B}<\left|\mathcal{I}_{\varepsilon}\right|^{2}
$$

Now one can use the relation between Feynman propagator and the Wightman function $i G_{F}\left(X_{j}, X_{j^{\prime}}\right)=G_{W}\left(X_{j}, X_{j^{\prime}}\right)+i G_{R}\left(X_{j^{\prime}}, X_{j}\right)=G_{W}\left(X_{j}, X_{j^{\prime}}\right)+\theta\left(T^{\prime}-T\right)\left\{G_{W}\left(X_{j^{\prime}}, X_{j}\right)-\right.$ $\left.G_{W}\left(X_{j}, X_{j^{\prime}}\right)\right\}$ to simplify the calculation of the integral $\mathcal{I}_{\varepsilon}$. In particular, one can now express that integral as

$$
\begin{aligned}
\mathcal{I}_{\varepsilon}= & -\int_{-\infty}^{\infty} d \tau_{B} \int_{-\infty}^{\infty} d \tau_{A} e^{i\left(\Delta E^{B} \tau_{B}+\Delta E^{A} \tau_{A}\right)} \times \\
& {\left[G_{W}\left(X_{B}, X_{A}\right)+\theta\left(T_{A}-T_{B}\right)\left\{G_{W}\left(X_{A}, X_{B}\right)-G_{W}\left(X_{B}, X_{A}\right)\right\}\right] . }
\end{aligned}
$$

We will use the above form for our purpose. It is observed that one only needs the expressions of the integrals $\mathcal{I}_{A}, \mathcal{I}_{B}$ and $\mathcal{I}_{\varepsilon}$ for verification of the condition (2.7) for entanglement harvesting. From eq. (2.4) and (2.8) we observe that all of these integrals can be evaluated in terms of the positive frequency Wightman functions.

When the condition for entanglement harvesting (2.7) is satisfied, it is convenient to study different entanglement measures. In this regard, one relevant entanglement measure is the negativity [52-55], which signifies the upper bound of the distillable entanglement and is obtained from the sum of all negative eigenvalues of the partial transpose of $\rho_{A B}$. In the two qubits case another important and more convenient entanglement measure is the concurrence $\mathcal{C}\left(\rho_{A B}\right)[38,40,49]$, from which entanglement of formation $E_{F}\left(\rho_{A B}\right)$ is estimated, see [38, 40, 56-58]. For two-qubits, the concurrence is given by, see [38],

$$
\begin{aligned}
\mathcal{C}\left(\rho_{A B}\right) & =\max \left[0,2 c^{2}\left(|\varepsilon|-\sqrt{P_{A} P_{B}}\right)+\mathcal{O}\left(c^{4}\right)\right] \\
& =\max \left[0,2 c^{2}\left|\left\langle E_{1}^{B}\left|m_{B}(0)\right| E_{0}^{B}\right\rangle\right|\left|\left\langle E_{1}^{A}\left|m_{A}(0)\right| E_{0}^{A}\right\rangle\right|\left(\left|\mathcal{I}_{\varepsilon}\right|-\sqrt{\mathcal{I}_{A} \mathcal{I}_{B}}\right)+\mathcal{O}\left(c^{4}\right)\right],
\end{aligned}
$$

where, an equal magnitude of the coupling constant $c_{A}=c_{B}=c$ between different detectors and the scalar field is assumed. It should be noted that the quantities $\left|\left\langle E_{1}^{j}\left|m_{j}(0)\right| E_{0}^{j}\right\rangle\right|$ are specified by the detectors' internal structure and do not take contributions from the considered spacetime and background scalar fields. Since we are interested to investigate the entanglement harvesting due to the motions of these detectors, then for a specific detector configuration it is only relevant to study the nature of

$$
\mathcal{C}_{\mathcal{I}}=\left(\left|\mathcal{I}_{\varepsilon}\right|-\sqrt{\mathcal{I}_{A} \mathcal{I}_{B}}\right)
$$

as far as concurrence is concerned. It should also be noted that in the symmetric case $\mathcal{I}_{A}=$ $\mathcal{I}_{B}$, which for example, can happen in the case of the equal magnitude of the acceleration of the two detectors, this relevant quantity signifying the concurrence is given by $\mathcal{C}_{\mathcal{I}}=$ $\left(\left|\mathcal{I}_{\varepsilon}\right|-\mathcal{I}_{j}\right)$, see $[38,40]$. In our later analysis, we shall be studying this $\mathcal{C}_{\mathcal{I}}$ to talk about the entanglement measure in our considered system. Particularly, by this, we will be investing the nature of entanglement harvesting for different parameters of our system. 
On the other hand, the total correlations, i.e., the entirety of classical and quantum correlations, between the two detectors $A$ and $B$ with the observers Alice and Bob is quantified by mutual information $\mathcal{M}$, defined as

$$
\mathcal{M}\left(\rho_{A B}\right) \equiv S\left(\rho_{A}\right)+S\left(\rho_{B}\right)-S\left(\rho_{A B}\right),
$$

where, $\rho_{A} \equiv \operatorname{Tr}_{B}\left(\rho_{A B}\right)$ and $\rho_{B} \equiv \operatorname{Tr}_{A}\left(\rho_{A B}\right)$ are the reduced density matrices corresponding to the detectors $A$ and $B$, and $S(\rho) \equiv-\operatorname{Tr}(\rho \ln \rho)$ is the von Neumann entropy corresponding to the state with $\rho$ to be the density matrix. Using the expression of the density matrix from eq. (2.2), and considering the equal couplings between the field and the two detectors, one can express the mutual information of (2.11) as [42]

$$
\mathcal{M}\left(\rho_{A B}\right)=c^{2}\left[P_{+} \ln P_{+}+P_{-} \ln P_{-}-P_{A} \ln P_{A}-P_{B} \ln P_{B}\right]+\mathcal{O}\left(c^{4}\right),
$$

where, the quantities $P_{ \pm}$are given by

$$
P_{ \pm}=\frac{1}{2}\left[P_{A}+P_{B} \pm \sqrt{\left(P_{A}-P_{B}\right)^{2}+4\left|P_{A B}\right|^{2}}\right] .
$$

We mention that one may encounter situations when both the concurrence and mutual information are not simultaneously non-zero for a specific system. Between the concurrence and mutual information, if only the latter is non-zero, the correlation is considered classical. Therefore, it is necessary to investigate both of these measures to understand the correlation between the two detectors.

\section{Accelerated observers in a thermal bath}

This section discusses the relevant coordinate systems for our accelerated observers. We realized that the whole analysis is more convenient under the decomposition of field modes in the Rindler frame and writing the Rindler annihilation and creation operators in terms of those of Unruh modes'. This will be introduced in a separate subsection. Finally, all the required positive frequency Wightman functions, both in $(1+1)$ and $(1+3)$ dimensions, for these fields will be evaluated with respect to the Minkowski vacuum, which is also the vacuum for Unruh modes.

\subsection{Coordinate systems}

The motion of a uniformly accelerated object is described by the Rindler coordinates which correspond to specific regions in the Minkowski spacetime, known as the Rindler wedges [59]. One can move to these Rindler coordinates from the flat Minkowski coordinates $(T, X, Y, Z)$ in $(3+1)$ dimensions, with the line element

$$
d s^{2}=-d T^{2}+d X^{2}+d Y^{2}+d Z^{2},
$$

by a coordinate transformation relating the time $T$ and the spatial direction in which the object is accelerated. Without loss of generality we consider that particular axis of acceleration to be along the Minkowski $X$ direction. Then the other two coordinates $(Y, Z)$ 
remain unchanged by the Rindler transformation. The transformations to the coordinates $(\eta, \xi)$ in the right Rindler wedge (RRW), i.e., the region $|T|<X$ in the Minkowski spacetime; and to $\left(\eta^{\prime}, \xi^{\prime}\right)$ in the left Rindler wedge (LRW), confined in a region $|T|<-X$ of the Minkowski spacetime, are

$$
\begin{array}{lll}
T=\frac{e^{a \xi}}{a} \sinh a \eta, & X=\frac{e^{a \xi}}{a} \cosh a \eta & \text { in RRW } \\
T=-\frac{e^{a \xi^{\prime}}}{a} \sinh a \eta^{\prime}, & X=-\frac{e^{a \xi^{\prime}}}{a} \cosh a \eta^{\prime} & \text { in LRW } .
\end{array}
$$

Both of these transformations lead to the same line-element corresponding to an accelerated observer in terms of the Rindler coordinates, expressed as

$$
d s^{2}=e^{2 a \xi}\left[-d \eta^{2}+d \xi^{2}\right]+d Y^{2}+d Z^{2} .
$$

One can perceive that these transformations in $(1+1)$ dimensions are trivially same as in that case the coordinates $Y$ and $Z$ cease to exist. In RRW and LRW one can estimate the proper times and proper accelerations to be

$$
\begin{aligned}
& \tau=e^{a \xi} \eta, \quad b=a e^{-a \xi} \quad \text { in RRW ; } \\
& \tau^{\prime}=-e^{a \xi^{\prime}} \eta^{\prime}, \quad b^{\prime}=a e^{-a \xi^{\prime}} \quad \text { in LRW . }
\end{aligned}
$$

Then the coordinate transformations (3.2) in terms of proper time and acceleration become

$$
\begin{aligned}
& T=\frac{1}{b} \sinh b \tau, \quad X=\frac{1}{b} \cosh b \tau \quad \text { in RRW; } \\
& T=\frac{1}{b^{\prime}} \sinh b^{\prime} \tau^{\prime}, \quad X=-\frac{1}{b^{\prime}} \cosh b^{\prime} \tau^{\prime} \quad \text { in LRW. }
\end{aligned}
$$

One can notice that $\eta,-\eta^{\prime}$ denote the proper times in RRW and LRW respectively while $a$ is the proper acceleration of the observer when $\xi=0=\xi^{\prime}$.

\subsection{Scalar field decomposition corresponding to an accelerated observer}

To address the situation of an accelerated observer in a thermal bath one can consider expressing the thermal two-point function in terms of the Minkowski modes and then make the Rindler coordinate transformation from eq. (3.5). However, the Green's function obtained in this way, for thermal field, is not time translational invariant in terms of proper time and a prescription to obtain unit time detector response using them is not possible [47]. On the other hand, one can also express the scalar field $\Phi(x)$ in terms of the Rindler modes and operators for which the corresponding vacuum is the Rindler vacuum. Then using the procedure as presented by Unruh in 1976 (see [26]), by transforming the Rindler operators to the Unruh operators which correspond to the vacuum of the Unruh modes (which is here Minkowski vacuum), one can construct Wightman function corresponding to accelerating observers in thermal Minkowski background. This way of construction provides the proper time translation invariance in a natural way and analysis becomes analytically more tractable (e.g. see [47]). 
The procedure of decomposing the scalar field in terms of the Unruh operators is elaborately discussed in $[60,61]$. Here we give a brief recollection of the construction and refer to the article [47] for further understandings. We first consider the case in $(1+1)$ dimensions and the $(1+3)$ dimensional result will follow accordingly. The equation of motion for a minimally coupled, massless free scalar field $\Phi$ is expressed as $\square \Phi=0$.

\subsection{1 (1+1) dimensions}

In terms of the Rindler coordinates in $(1+1)$ dimensions this equation has solutions, suggesting set of modes in the right and left Rindler wedges as [60,61]

$$
\begin{aligned}
{ }^{R} u_{k} & =\frac{1}{\sqrt{4 \pi \omega}} e^{i k \xi-i \omega \eta} & & \text { in RRW } \\
& =0 & & \text { in LRW } \\
{ }^{L} u_{k} & =\frac{1}{\sqrt{4 \pi \omega}} e^{i k \xi+i \omega \eta} & & \text { in LRW } \\
& =0 & & \text { in RRW. }
\end{aligned}
$$

The scalar field is expressed in terms of the Rindler modes and operators, see [60], as $\Phi(X)=\sum_{k=-\infty}^{\infty}\left[b_{k}^{R} R u_{k}+b_{k}^{R^{\dagger}} R_{u_{k}^{*}}+b_{k}^{L}{ }^{L} u_{k}+b_{k}^{L^{\dagger}}{ }^{L} u_{k}^{*}\right]$, where superscript $L$ and $R$ correspond to the left and the right Rindler wedges respectively, and the annihilation operators annihilate the Rindler vacuum $\left|0_{\mathcal{R}}\right\rangle$, i.e. $b_{k}^{R}\left|0_{\mathcal{R}}\right\rangle=0=b_{k}^{L}\left|0_{\mathcal{R}}\right\rangle$. In the right or left Rindler wedges where the field modes ${ }^{L} u_{k}=0$ or ${ }^{R} u_{k}=0$, the scalar field takes the form

$$
\begin{aligned}
& \Phi^{R}(X)=\sum_{k=-\infty}^{\infty}\left[b_{k}^{R} R u_{k}+b_{k}^{R^{\dagger}} R u_{k}^{*}\right], \\
& \text { or } \Phi^{L}(X)=\sum_{k=-\infty}^{\infty}\left[b_{k}^{L}{ }^{L} u_{k}+b_{k}^{L^{\dagger}}{ }^{L} u_{k}^{*}\right] \text {. }
\end{aligned}
$$

One can use this scalar field decomposition in terms of the Rindler modes and operators to obtain a two-point function corresponding to an accelerated observer in Minkowski vacuum. Here, it should be noted that the operators $b_{k}^{R}$ and $b_{k}^{L}$ in eq. (3.7) do not annihilate the Minkowski vacuum, and the operations of the Rindler ladder operators on the Minkowski vacuum is obtained from the cumbersome calculations of Bogoliubov transformation. However, there is a simpler way out of this situation as provided by Unruh [26], where he prescribed field modes out of these Rindler modes which are analytic in the whole region of the Minkowski spacetime. These Unruh modes have the positive frequency analyticity property with respect to the Minkowski time, same as the Minkowski modes. This enables one to decompose the scalar field in terms of these Unruh modes and operators, which annihilate the Minkowski vacuum. The Unruh modes are obtained from the combination of the Rindler modes ${ }^{R} u_{k}+e^{-\pi \omega / a} L_{u_{-k}^{*}}$ and ${ }^{R} u_{-k}^{*}+e^{\pi \omega / a} L_{u_{k}}$, see [60]. In terms of the Unruh modes and operators the scalar field is expressed as [60]

$\Phi(X)=\sum_{k=-\infty}^{\infty} \frac{1}{\sqrt{2 \sinh \frac{\pi \omega}{a}}}\left[d_{k}^{1}\left(e^{\frac{\pi \omega}{2 a} R} u_{k}+e^{-\frac{\pi \omega}{2 a} L} u_{-k}^{*}\right)+d_{k}^{2}\left(e^{-\frac{\pi \omega}{2 a} R} u_{-k}^{*}+e^{\frac{\pi \omega}{2 a} L} u_{k}\right)\right]+$ h.c. 
where h.c. stands for Hermitian conjugate. The Unruh annihilation operators annihilate the Minkowski vacuum $d_{k}^{1}\left|0_{M}\right\rangle=d_{k}^{2}\left|0_{M}\right\rangle=0$. To obtain the positive frequency Green's function using the field decompositions of eq. (3.7), one needs a transformation between the Rindler operators and the Unruh operators, see [60], which is

$$
\begin{aligned}
& b_{k}^{L}=\frac{1}{\sqrt{2 \sinh \frac{\pi \omega}{a}}}\left[e^{\frac{\pi \omega}{2 a}} d_{k}^{2}+e^{-\frac{\pi \omega}{2 a}} d_{-k}^{1^{\dagger}}\right] \\
& b_{k}^{R}=\frac{1}{\sqrt{2 \sinh \frac{\pi \omega}{a}}}\left[e^{\frac{\pi \omega}{2 a}} d_{k}^{1}+e^{-\frac{\pi \omega}{2 a}} d_{-k}^{2^{\dagger}}\right],
\end{aligned}
$$

and, it is similar to the Bogoliubov transformation. Then putting this transformation in eq. (3.7) one can get the expression of the field in the RRW and LRW in terms of the Unruh operators as

$$
\begin{aligned}
& \Phi^{R}(X)=\sum_{k=-\infty}^{\infty} \frac{1}{\sqrt{2 \sinh \frac{\pi \omega}{a}}}\left[d_{k}^{1} e^{\frac{\pi \omega}{2 a} R} u_{k}+d_{k}^{2} e^{-\frac{\pi \omega}{2 a} R} u_{-k}^{*}\right]+\text { h.c. }, \\
& \Phi^{L}(X)=\sum_{k=-\infty}^{\infty} \frac{1}{\sqrt{2 \sinh \frac{\pi \omega}{a}}}\left[d_{k}^{1} e^{-\frac{\pi \omega}{2 a}} L_{u_{-k}^{*}}+d_{k}^{2} e^{\frac{\pi \omega}{2 a}} L u_{k}\right]+\text { h.c. . }
\end{aligned}
$$

Now these expression of the scalar fields in RRW and LRW can be used to obtain the expressions of the positive frequency Green's function corresponding to accelerated observers in thermal bath.

\subsection{2 (1+3) dimensions}

Like the above analysis, in $(1+3)$ dimensions also, one can proceed in a similar manner to get the Scalar field in terms of the Unruh operators. In particular, from the equation of motion $\square \Phi=0$ one can get the Rindler modes in the right and the left Rindler wedges as

$$
\begin{aligned}
R_{u_{\omega, k_{p}}} & =\frac{1}{2 \pi^{2}} \sqrt{\frac{\sinh \left(\frac{\pi \omega}{a}\right)}{a}} \mathcal{K}\left[\frac{i \omega}{a}, \frac{\left|k_{p}\right| e^{a \xi}}{a}\right] e^{-i \omega \eta+i \overrightarrow{k_{p} \cdot \vec{x}}} & & \text { in RRW } \\
& =0 & & \text { in LRW } \\
{ }^{L} u_{\omega, k_{p}} & =\frac{1}{2 \pi^{2}} \sqrt{\frac{\sinh \left(\frac{\pi \omega}{a}\right)}{a}} \mathcal{K}\left[\frac{i \omega}{a}, \frac{\left|k_{p}\right| e^{a \xi}}{a}\right] e^{i \omega \eta+i \overrightarrow{k_{p} \cdot \vec{x}}} & & \text { in LRW } \\
& =0 & & \text { in RRW }
\end{aligned}
$$

where, $\mathcal{K}[n, z]$ denotes the modified Bessel function of the second kind of order $n, \vec{x}$ is perpendicular to the direction of acceleration, i.e., in the $Y-Z$ plane, see [59, 62, 63], and $\overrightarrow{k_{p}}$ denotes the transverse wave vector in the $Y-Z$ plane. Like the $(1+1)$ dimensional case here also one can construct the Unruh modes [59] out of the Rindler modes, which are analytic in the whole Minkowski spacetime and gives positive frequency mode solutions with respect to the Minkowski time. Then in $(1+3)$ dimensions the scalar field in the RRW and LRW using the Unruh operators, see [47, 59] for a detailed description, can be 
expressed in forms

$$
\begin{aligned}
& \Phi^{R}(X)=\sum_{\omega=0}^{\infty} \sum_{k_{p}=-\infty}^{\infty} \frac{1}{\sqrt{2 \sinh \frac{\pi \omega}{a}}}\left[d_{\omega, k_{p}}^{1} e^{\frac{\pi \omega}{2 a} R} u_{\omega, k_{p}}+d_{\omega, k_{p}}^{2} e^{-\frac{\pi \omega}{2 a} R} u_{\omega,-k_{p}}^{*}\right]+\text { h.c. } \\
& \Phi^{L}(X)=\sum_{\omega=0}^{\infty} \sum_{k_{p}=-\infty}^{\infty} \frac{1}{\sqrt{2 \sinh \frac{\pi \omega}{a}}}\left[d_{\omega, k_{p}}^{1} e^{-\frac{\pi \omega}{2 a} L} u_{\omega,-k_{p}}^{*}+d_{\omega, k_{p}}^{2} e^{\frac{\pi \omega}{2 a} L} u_{\omega, k_{p}}\right]+\text { h.c. }
\end{aligned}
$$

This is exactly same as the $(1+1)$ dimensional expression with the Rindler field modes ${ }^{R} u_{\omega, k_{p}}$ and ${ }^{L} u_{\omega, k_{p}}$ are now given by different expressions, and the sum is now on $\omega$ and two components of $k_{p}$ rather than one wave vector $k$ of the $(1+1)$ dimensional case.

\subsection{Two-point correlators for thermal field}

Considering a scalar field $\Phi(X)=\Phi(T, \mathbf{X})$ in equilibrium with a thermal bath of temperature $T^{(f)}=1 /\left(k_{B} \beta\right)$, where $k_{B}$ is the Boltzmann constant, the thermal Green's (Wightman) function can be obtained by taking Gibbs ensemble average of the operator $\Phi\left(X_{2}\right) \Phi\left(X_{1}\right)$ as

$$
\begin{aligned}
G^{\beta}\left(X_{2} ; X_{1}\right) & =\left\langle\Phi\left(X_{2}\right) \Phi\left(X_{1}\right)\right\rangle_{\beta} \\
& =\frac{1}{Z} \operatorname{Tr}\left[e^{-\beta H} \Phi\left(X_{2}\right) \Phi\left(X_{1}\right)\right],
\end{aligned}
$$

where, $X_{1}$ and $X_{2}$ are two events in the spacetime, $Z=\operatorname{Tr}[\exp (-\beta H)]$ denotes the partition function, and $H$ denotes the Hamiltonian of free massless scalar field.

\subsection{1 (1+1) dimensions}

In $(1+1)$ dimensions to obtain the thermal Green's function corresponding to accelerated observers, with respect to Rindler modes, we consider massless scalar field where $\omega=\omega_{k}=$ $|k|$. The Hamiltonian related to the $k^{t h}$ excitation corresponding to the Unruh operators, which respect the Unruh vacuum, is $H_{k}=\left(d_{k}^{1^{\dagger}} d_{k}^{1}+d_{k}^{2^{\dagger}} d_{k}^{2}\right) \omega_{k}$. Then the thermal Green's function, defined by eq. (3.13), corresponding to an accelerated observer, see [47], can be expressed as

$$
\begin{aligned}
& G_{W_{R}}^{\beta}\left(\Delta \xi_{j l}, \Delta \eta_{j l}\right)= \\
& \int_{-\infty}^{\infty} \frac{d k}{8 \pi \omega_{k} \sqrt{\sinh \frac{\pi \omega_{k}}{a_{j}} \sinh \frac{\pi \omega_{k}}{a_{l}}}} \times \\
& {\left[\frac{1}{1-e^{-\beta \omega_{k}}}\left\{e^{i k \Delta \xi_{j l}-i \omega_{k} \Delta \eta_{j l} l} e^{\frac{\pi \omega_{k}}{2}\left(\frac{1}{a_{j}}+\frac{1}{a_{l}}\right)}+e^{i k \Delta \xi_{j l}+i \omega_{k} \Delta \eta_{j l}} e^{-\frac{\pi \omega_{k}}{2}\left(\frac{1}{a_{j}}+\frac{1}{a_{l}}\right)}\right\}\right.} \\
& \left.+\frac{1}{e^{\beta \omega_{k}}-1}\left\{e^{-i k \Delta \xi_{j l}+i \omega_{k} \Delta \eta_{j l}} e^{\frac{\pi \omega_{k}}{2}\left(\frac{1}{a_{j}}+\frac{1}{a_{l}}\right)}+e^{-i k \Delta \xi_{j l}-i \omega_{k} \Delta \eta_{j l}} e^{-\frac{\pi \omega_{k}}{2}\left(\frac{1}{a_{j}}+\frac{1}{a_{l}}\right)}\right\}\right],
\end{aligned}
$$

where, $j, l$ denote different detectors, and $\Delta \xi_{j l}=\xi_{j, 2}-\xi_{l, 1}, \Delta \eta_{j l}=\eta_{j, 2}-\eta_{l, 1}$. For observers in the left Rindler wedge immersed in a thermal bath the Wightman function $G_{W_{L}}^{\beta}\left(\Delta \xi_{j l}, \Delta \eta_{j l}\right)$ is obtained from the expression of eq. (3.14) with $\Delta \eta_{j l} \rightarrow-\Delta \eta_{j l}$. 
Similarly for observers with one in the right Rindler wedge and one in the left Rindler wedge immersed in a thermal bath the Wightman function can be expressed, using the appropriate field modes from eq. (3.10), as

$$
\begin{aligned}
& G_{W_{L R}}^{\beta}\left(\Delta \xi_{j l}, \Delta \eta_{j l}\right)= \\
& \int_{-\infty}^{\infty} \frac{d k}{8 \pi \omega_{k} \sqrt{\sinh \frac{\pi \omega_{k}}{a_{j}} \sinh \frac{\pi \omega_{k}}{a_{l}}}} \times \\
& {\left[\frac{1}{1-e^{-\beta \omega_{k}}}\left\{e^{i k \Delta \xi_{j l}-i \omega_{k} \Delta \eta_{j l}} e^{-\frac{\pi \omega_{k}}{2}\left(\frac{1}{a_{j}}-\frac{1}{a_{l}}\right)}+e^{i k \Delta \xi_{j l}+i \omega_{k} \Delta \eta_{j l}} e^{\frac{\pi \omega_{k}}{2}\left(\frac{1}{a_{j}}-\frac{1}{a_{l}}\right)}\right\}\right.} \\
& \left.+\frac{1}{e^{\beta \omega_{k}}-1}\left\{e^{-i k \Delta \xi_{j l}+i \omega_{k} \Delta \eta_{j l}} e^{-\frac{\pi \omega_{k}}{2}\left(\frac{1}{a_{j}}-\frac{1}{a_{l}}\right)}+e^{-i k \Delta \xi_{j l}-i \omega_{k} \Delta \eta_{j l}} e^{\frac{\pi \omega_{k}}{2}\left(\frac{1}{a_{j}}-\frac{1}{a_{l}}\right)}\right\}\right],
\end{aligned}
$$

where, we have considered the $j^{\text {th }}$ detector to be in the left Rindler wedge and the detector denoted by $l$ is in the right Rindler wedge. We also mention that the Wightman function $G_{W_{R L}}^{\beta}\left(\Delta \xi_{j l}, \Delta \eta_{j l}\right)$, where the detectors denoted by $j$ and $l$ are in right and left Rindler wedges is obtained from the complex conjugate of the expression in the right hand side of eq. (3.15). It should be noted that from eq. (3.14) the thermal Green's function corresponding to a single accelerated detector can also be obtained by making $a_{j}=a_{l}$.

\subsection{2 (1+3) dimensions}

One can obtain the thermal Green's function corresponding to accelerated observers, with respect to Rindler modes in $(1+3)$ dimensions in a similar manner. The field decomposition is taken from eq. (3.12) and the Hamiltonian corresponding to the Unruh operators is $H_{\omega, k_{p}}=\left(d_{\omega, k_{p}}^{1^{\dagger}} d_{\omega, k_{p}}^{1}+d_{\omega, k_{p}}^{2^{\dagger}} d_{\omega, k_{p}}^{2}\right) \omega$. Then in RRW the Green's function corresponding to an accelerated observer in thermal bath [47] is

$$
\begin{aligned}
G_{W_{R}^{3 D}}^{\beta}\left(\Delta \eta_{j l}\right)= & \int_{0}^{\infty} d \omega \int \frac{d^{2} k_{p}}{(2 \pi)^{4}} \frac{2}{\sqrt{a_{j} a_{l}}} \\
& {\left[\frac{e^{-i \omega \Delta \eta_{j l}} e^{\frac{\pi \omega}{2}\left(\frac{1}{a_{j}}+\frac{1}{a_{l}}\right)}+e^{i \omega \Delta \eta_{j l}} e^{-\frac{\pi \omega}{2}\left(\frac{1}{a_{j}}+\frac{1}{a_{l}}\right)}}{1-e^{-\beta \omega}}\right.} \\
& \left.+\frac{e^{i \omega \Delta \eta_{j l}} e^{\frac{\pi \omega}{2}\left(\frac{1}{a_{j}}+\frac{1}{a_{l}}\right)}+e^{-i \omega \Delta \eta_{j l} l} e^{-\frac{\pi \omega}{2}\left(\frac{1}{a_{j}}+\frac{1}{a_{l}}\right)}}{e^{\beta \omega}-1}\right] \\
& \mathcal{K}\left[\frac{i \omega}{a_{j}}, \frac{\left|k_{p}\right| e^{a_{j} \xi_{j}}}{a_{j}}\right] \mathcal{K}\left[\frac{i \omega}{a_{l}}, \frac{\left|k_{p}\right| e^{a_{l} \xi_{l}}}{a_{l}}\right],
\end{aligned}
$$

where, $\Delta \eta_{j l}=\eta_{j, 2}-\eta_{l, 1}$ and $\xi_{j}$ is the fixed Rindler spatial coordinate corresponding to the $j^{\text {th }}$ detector. It should be noted that the above Green's function is time translational invariant. 
On the other hand, the Wightman function corresponding to two observers with anti parallel acceleration is

$$
\begin{aligned}
G_{W_{L R}^{3 D}}^{\beta}\left(\Delta \eta_{j l}\right)= & \int_{0}^{\infty} d \omega \int \frac{d^{2} k_{p}}{(2 \pi)^{4}} \frac{2}{\sqrt{a_{j} a_{l}}} \\
& {\left[\frac{e^{-i \omega \Delta \eta_{j l}} e^{-\frac{\pi \omega}{2}\left(\frac{1}{a_{j}}-\frac{1}{a_{l}}\right)}+e^{i \omega \Delta \eta_{j l}} e^{\frac{\pi \omega}{2}\left(\frac{1}{a_{j}}-\frac{1}{a_{l}}\right)}}{1-e^{-\beta \omega}}\right.} \\
& \left.+\frac{e^{i \omega \Delta \eta_{j l}} e^{-\frac{\pi \omega}{2}\left(\frac{1}{a_{j}}-\frac{1}{a_{l}}\right)}+e^{-i \omega \Delta \eta_{j l}} e^{\frac{\pi \omega}{2}\left(\frac{1}{a_{j}}-\frac{1}{a_{l}}\right)}}{e^{\beta \omega}-1}\right] \\
& \mathcal{K}\left[\frac{i \omega}{a_{j}}, \frac{\left|k_{p}\right| e^{a_{j} \xi_{j}}}{a_{j}}\right] \mathcal{K}\left[\frac{i \omega}{a_{l}}, \frac{\left|k_{p}\right| e^{a_{l} \xi_{l}}}{a_{l}}\right] .
\end{aligned}
$$

Here also $j$ and $l$ denote detectors in left and in right Rindler wedges, and the Wightman function $G_{W_{R L}^{3 D}}^{\beta}\left(\Delta \xi_{j l}, \Delta \eta_{j l}\right)$, with $j$ and $l$ denoting detectors in right and left Rindler wedges, is obtained from the complex conjugate of the expression (3.17). The thermal Green's function corresponding to a single accelerated detector can be obtained by making $a_{j}=a_{l}$ in eq. (3.16).

Having equipped with all the necessary results we will next investigate the role of temperature of the field on the entanglement harvesting between the two uniformly accelerated detectors. We will have particular interest here on two situations - (i) both the detectors are in right wedge and (ii) one is in right wedge and another one is in left wedge. This will be done in the next section.

\section{Entanglement harvesting}

In this section we investigate the condition of entanglement extraction from eq. (2.7) for accelerated detectors in parallel or anti-parallel relative motion in a thermal bath. In particular, we aim to understand the effects of the thermal bath in addition to the acceleration on this entanglement harvesting condition. We shall also be looking into the entanglement measure, namely the concurrence, for the aforementioned observers. In this regard, we first estimate the quantities $\mathcal{I}_{j}\left(\Delta E^{j}\right)$ for the detectors accelerated in right or in left Rindler wedge. These are common quantities for both parallel and anti-parallel situations.

We first consider the $(1+1)$ dimensional case. For an observer accelerated in the right Rindler wedge we take the expression of the Wightman function from eq. (3.14) with equal acceleration. Then in RRW one can estimate the integral $\mathcal{I}_{j}\left(\Delta E^{j}\right)$ as

$$
\begin{aligned}
\mathcal{I}_{j}^{R}\left(\Delta E^{j}\right) & =\frac{1}{2} \int_{-\infty}^{\infty} d v_{j} \int_{-\infty}^{\infty} d u_{j} e^{-i \Delta E^{j} u_{j}} G_{W_{R}}^{\beta}\left(u_{j}\right) \\
& =\delta(0) \frac{\pi}{2 \Delta E^{j} a_{j}} \frac{1}{\sinh \frac{\pi \Delta E^{j}}{a_{j}}}\left[\frac{e^{-\frac{\pi \Delta E^{j}}{a_{j}}}}{1-e^{-\beta \Delta E^{j}}}+\frac{e^{\frac{\pi \Delta E_{j}^{j}}{a_{j}}}}{e^{\beta \Delta E^{j}}-1}\right],
\end{aligned}
$$


where we have used $\eta_{j}^{\prime}=\tau_{j}^{\prime}, \eta_{j}=\tau_{j}$ for $\xi_{j}^{\prime}=0=\xi_{j}$ as observed from eq. (3.4). We have considered the change of variables $v_{j}=\tau_{j}^{\prime}+\tau_{j}, u_{j}=\tau_{j}^{\prime}-\tau_{j}$. In the above expression the Dirac delta distribution is obtained from $\delta(0)=(1 / 2 \pi) \int_{-\infty}^{\infty} d \gamma_{j}$, where $\gamma_{j}=a_{j} v_{j}$ is a dimensionless parameter characterizing the proper time of the detector. On the other hand, in a similar manner in LRW also one can estimate the integral $\mathcal{I}_{j}^{L}\left(\Delta E^{j}\right)$ using the complex conjugate of the Wightman function from eq. (3.14) and the relation between the Rindler time and the detector proper times $\eta_{j}^{\prime}=-\tau_{j}^{\prime}, \eta_{j}=-\tau_{j}$ in LRW for $\xi_{j}^{\prime}=0=\xi_{j}$ from (3.4). In $(1+1)$ dimensions this expression comes out to be the same as the one obtained for the observer in RRW (4.1), i.e., we get $\mathcal{I}_{j}^{L}\left(\Delta E^{j}\right)=\mathcal{I}_{j}^{R}\left(\Delta E^{j}\right)$.

Similarly, in $(1+3)$ dimensions also one can find out the quantities $\mathcal{I}_{j}^{R}\left(\Delta E^{j}\right)$ and $\mathcal{I}_{j}^{L}\left(\Delta E^{j}\right)$. In particular, these quantities in right and left Rindler wedges are given by the same expression $\mathcal{I}_{j}^{R}\left(\Delta E^{j}\right)=\mathcal{I}_{j}^{L}\left(\Delta E^{j}\right)=\mathcal{I}_{j_{3 D}}\left(\Delta E^{j}\right)$. With the help of the Wightman function from eq. (3.16) this expression can be provided as

$$
\begin{aligned}
\mathcal{I}_{j_{3 D}}\left(\Delta E^{j}\right) & =\frac{1}{2} \int_{-\infty}^{\infty} d v_{j} \int_{-\infty}^{\infty} d u_{j} e^{-i \Delta E^{j} u_{j}} G_{W_{3 D}}^{\beta}\left(u_{j}\right) \\
& =\delta(0) \frac{1}{2 \pi a_{j}^{2}}\left[\frac{e^{-\frac{\pi \Delta E^{j}}{a_{j}}}}{1-e^{-\beta \Delta E^{j}}}+\frac{e^{\frac{\pi \Delta E^{j}}{a_{j}}}}{e^{\beta \Delta E^{j}}-1}\right] \Upsilon\left(\Delta E^{j}, a_{j}, a_{j}\right),
\end{aligned}
$$

where, in this case the quantity $\Upsilon\left(\Delta E^{j}, a_{j}, a_{j}\right)=\pi a_{j} \Delta E^{j} /\left(2 \sinh \left(\pi \Delta E^{j} / a_{j}\right)\right)$, and it is obtained from a general expression of integral

$$
\Upsilon\left(\bar{\varepsilon}, a_{j}, a_{l}\right)=\int_{0}^{\infty} k_{p} d k_{p} \mathcal{K}\left[\frac{i \bar{\varepsilon}}{a_{j}}, \frac{k_{p}}{a_{j}}\right] \mathcal{K}\left[\frac{i \bar{\varepsilon}}{a_{l}}, \frac{k_{p}}{a_{l}}\right] .
$$

Now it should be noted that the integrals representing transition probabilities from eq. (4.1), and (4.2) can be multiplied on both sides by $\Delta E_{j}^{2}$ to make them dimensionless. In this regard, we define other dimensionless parameters of the system as

$$
\alpha_{j}=\frac{a_{j}}{\Delta E_{j}} ; \quad \sigma_{j}=\beta \Delta E_{j} .
$$

It will be much more convenient to represent the necessary diagrams in our subsequent analysis with respect to these dimensionless parameters and quantities. In our subsequent analysis we specifically consider the situation of two observers accelerated parallelly or anti-parallelly in a thermal bath, and in particular, going to estimate the integrals $\mathcal{I}_{\varepsilon}$. Then we shall analyze the condition of eq. (2.7), and verify the possibility of entanglement extraction in those specific cases.

It should also be noted that for the verification of the condition (2.7) it is imperative to evaluate the expression of $\mathcal{I}_{\varepsilon}$. From eq. (2.8) it is observed that the expression of $\mathcal{I}_{\varepsilon}$ can be represented it terms of one quantity containing Wightman function and another one containing the Retarded Green's function. This second integral containing the Retarded Green's function also has a Heaviside step function $\theta\left(T_{A}-T_{B}\right)$ in it, which emerged from the representation of the Feynman propagator in terms of the Wightman functions. In a spacetime where the Wightman functions are constructed considering positive frequency 
modes functions defined with respect to time $t_{j}$, this step function shall become $\theta\left(t_{A}-\right.$ $t_{B}$ ). From eq. (3.14) to (3.17) all the Wightman functions are constructed using positive frequency Rindler modes of (3.6) and (3.11). Then with $\xi_{j}^{\prime}=0=\xi_{j}$ in right Rindler wedge $t_{j}=t_{j}^{\mathcal{R}}=\eta_{j}=\tau_{j}$ and in left Rindler wedge $t_{j}=t_{j}^{\mathcal{R}}=-\eta_{j}=\tau_{j}$. We shall be explicitly using these relations in the evaluation of the quantity $\mathcal{I}_{\varepsilon}$ in our subsequent analysis.

\subsection{Parallel acceleration: no harvesting}

In this subsection we consider the two observers Alice and Bob to be accelerated parallelly. We consider them to have the proper accelerations $a_{A}$ and $a_{B}$ and both of them to be in the right Rindler wedge. For convenience of calculation, in this case we express integral $\mathcal{I}_{\varepsilon}$ from eq. (2.8) as

$$
\begin{aligned}
\mathcal{I}_{\varepsilon}= & -\int_{-\infty}^{\infty} d \tau_{B} \int_{-\infty}^{\infty} d \tau_{A} e^{i\left(\Delta E^{B} \tau_{B}+\Delta E^{A} \tau_{A}\right)} \times \\
& {\left[G_{W}\left(X_{B}, X_{A}\right)+\theta\left(t_{A}^{\mathcal{R}}-t_{B}^{\mathcal{R}}\right)\left\{G_{W}\left(X_{A}, X_{B}\right)-G_{W}\left(X_{B}, X_{A}\right)\right\}\right] } \\
= & \mathcal{I}_{\varepsilon}^{W}+\mathcal{I}_{\varepsilon}^{R}
\end{aligned}
$$

Here the first integral $\mathcal{I}_{\varepsilon}^{W}$ contains the Wightman function, while the second integral $\mathcal{I}_{\varepsilon}^{R}$ represents the contribution of the retarded Green's function. We shall be using this expression to evaluate the integral $\mathcal{I}_{\varepsilon}$ separately in $(1+1)$ and $(1+3)$ dimensions in our following studies.

\subsection{1 (1+1) dimensions}

For the evaluation of $\mathcal{I}_{\varepsilon}^{W}$ and $\mathcal{I}_{\varepsilon}^{R}$ in $(1+1)$ dimensions we consider the positive frequency Wightman function (3.14). In particular, in the expression of this Green's function the indices $j$ and $l$ correspond to the detector $A$ and $B$ respectively. The relation between Rindler times and detector proper times are $\eta_{A}=\tau_{A}$ and $\eta_{B}=\tau_{B}$, considering $\xi_{A}=0=\xi_{B}$, i.e., assuming the accelerating detectors to be fixed at the origin of the respective Rindler frames, while the proper accelerations are $b_{j}=a_{j}$. Then the first integral $\mathcal{I}_{\varepsilon}^{W}$ can be expressed as

$$
\begin{aligned}
\mathcal{I}_{\varepsilon}^{W}= & -\int_{-\infty}^{\infty} d \tau_{B} \int_{-\infty}^{\infty} d \tau_{A} e^{i\left(\Delta E^{B} \tau_{B}+\Delta E^{A} \tau_{A}\right)} G_{W_{R}}^{\beta}\left(X_{B}, X_{A}\right) \\
= & -\delta\left(\frac{\Delta E^{A}+\Delta E^{B}}{\sqrt{a_{A} a_{B}}}\right) \frac{\pi}{\Delta \breve{E} \sqrt{a_{A} a_{B}}} \frac{1}{\sqrt{\sinh \frac{\pi \Delta \breve{E}}{a_{B}} \sinh \frac{\pi \Delta \breve{E}}{a_{A}}}} \\
& {\left[\frac{e^{\frac{\pi \Delta \breve{E}}{2}\left(\frac{1}{a_{B}}+\frac{1}{a_{A}}\right)}}{1-e^{-\beta \Delta \breve{E}}}+\frac{e^{-\frac{\pi \Delta \breve{E}}{2}\left(\frac{1}{a_{B}}+\frac{1}{a_{A}}\right)}}{e^{\beta \Delta \breve{E}}-1}\right], }
\end{aligned}
$$

where the expression of $\Delta \breve{E}$ is given by $\Delta \breve{E}=\left(\Delta E^{B}-\Delta E^{A}\right) / 2$. For the evaluation of this integral we have considered a change of variables $\tilde{v}=\tau_{B}+\tau_{A}$ and $\tilde{u}=\tau_{B}-\tau_{A}$. The Jacobian of this transformation from $\tau_{j}$ to $v$ and $u$ is $1 / 2$. On the other hand, using the 
same Wightman function from eq. (3.14) we get the integral $\mathcal{I}_{\varepsilon}^{R}$ to be

$$
\begin{aligned}
\mathcal{I}_{\varepsilon}^{R} & =-\int_{-\infty}^{\infty} d \tau_{B} \int_{-\infty}^{\infty} d \tau_{A} e^{i\left(\Delta E^{B} \tau_{B}+\Delta E^{A} \tau_{A}\right)} \theta\left(\tau_{A}-\tau_{B}\right)\left\{G_{W_{R}}^{\beta}\left(X_{A}, X_{B}\right)-G_{W_{R}}^{\beta}\left(X_{B}, X_{A}\right)\right\} \\
& =-\delta\left(\frac{\Delta E^{A}+\Delta E^{B}}{\sqrt{a_{A} a_{B}}}\right) \mathscr{I}_{1}^{R},
\end{aligned}
$$

which also contains a Dirac delta distribution, and $\mathscr{I}_{1}^{R}$ is given by

$$
\mathscr{I}_{1}^{R}=\int_{0}^{\infty} \frac{d \omega}{\omega \sqrt{a_{A} a_{B}}} \frac{\sinh \left[\frac{\pi \omega}{2}\left(\frac{1}{a_{B}}+\frac{1}{a_{A}}\right)\right]}{\sqrt{\sinh \frac{\pi \omega}{a_{B}} \sinh \frac{\pi \omega}{a_{A}}}} \int_{0}^{\infty} e^{-i \tilde{u} \Delta \breve{E}}\left(e^{-i \omega \tilde{u}}-e^{i \omega \tilde{u}}\right) .
$$

In the integrals (4.6) and (4.7) we have encountered expression $\delta\left(\left(\Delta E^{B}+\Delta E^{A}\right) / \sqrt{a_{A} a_{B}}\right)$, which definitely cannot give non-zero contribution as $\Delta E^{j}>0$. Then we have $\mathcal{I}_{\varepsilon}=0$ due to the contributions of $\mathcal{I}_{\varepsilon}^{W}$ and $\mathcal{I}_{\varepsilon}^{R}$. Whereas from eq. (4.1) we observe that $\mathcal{I}_{j}$ always are non-zero and also have a multiplicative $\delta(0)$. This signifies that for two observers accelerated parallelly the condition for entanglement harvesting (2.7) is not satisfied in $(1+1)$ dimensions.

\subsection{2 (1+3) dimensions}

We consider the positive frequency Wightman function (3.16) for the estimation of the quantities $\mathcal{I}_{\varepsilon}^{W}$ and $\mathcal{I}_{\varepsilon}^{R}$ in $(1+3)$ dimensions. Furthermore, we have identified the indices $j$ and $l$ with the detectors $A$ and $B$ respectively. Then proceeding like the earlier way the first integral $\mathcal{I}_{\varepsilon}^{W}$ becomes

$$
\begin{aligned}
\mathcal{I}_{\varepsilon}^{W}= & -\int_{-\infty}^{\infty} d \tau_{B} \int_{-\infty}^{\infty} d \tau_{A} e^{i\left(\Delta E^{B} \tau_{B}+\Delta E^{A} \tau_{A}\right)} G_{W_{R}^{3 D}}^{\beta}\left(X_{B}, X_{A}\right) \\
= & -\delta\left(\frac{\Delta E^{A}+\Delta E^{B}}{\sqrt{a_{A} a_{B}}}\right) \frac{1}{\pi a_{A} a_{B}} \Upsilon\left(\Delta \breve{E}, a_{A}, a_{B}\right) \\
& {\left[\frac{e^{\frac{\pi \Delta \breve{E}}{2}\left(\frac{1}{a_{B}}+\frac{1}{a_{A}}\right)}}{1-e^{-\beta \Delta \breve{E}}}+\frac{e^{-\frac{\pi \Delta \breve{E}}{2}\left(\frac{1}{a_{B}}+\frac{1}{a_{A}}\right)}}{e^{\beta \Delta \breve{E}}-1}\right] }
\end{aligned}
$$

Using the Wightman function (3.16) with the proper identification of the indices $j$ and $l$ to the detectors $A$ and $B$, we get the second part of the integral $\mathcal{I}_{\varepsilon}$ to be

$$
\begin{aligned}
\mathcal{I}_{\varepsilon}^{R} & =-\int_{-\infty}^{\infty} d \tau_{B} \int_{-\infty}^{\infty} d \tau_{A} e^{i\left(\Delta E^{B} \tau_{B}+\Delta E^{A} \tau_{A}\right)} \theta\left(\tau_{A}-\tau_{B}\right)\left\{G_{W_{R}^{3 D}}^{\beta}\left(X_{A}, X_{B}\right)-G_{W_{R}^{3 D}}^{\beta}\left(X_{B}, X_{A}\right)\right\} \\
& =-\delta\left(\frac{\Delta E^{B}+\Delta E^{A}}{\sqrt{a_{A} a_{B}}}\right) \mathscr{I}_{2}^{R}
\end{aligned}
$$

where, the expression of $\mathscr{I}_{2}^{R}$ is given by

$$
\mathscr{I}_{2}^{R}=\int_{0}^{\infty} \frac{d \omega}{\pi^{2}} \frac{\sinh \left[\frac{\pi \omega}{2}\left(\frac{1}{a_{B}}+\frac{1}{a_{A}}\right)\right]}{a_{A} a_{B}} \Upsilon\left(\Delta \breve{E}, a_{A}, a_{B}\right) \int_{0}^{\infty} e^{-i \tilde{u} \Delta \breve{E}}\left(e^{-i \omega \tilde{u}}-e^{i \omega \tilde{u}}\right)
$$


Here also in both of the integrals from (4.9) and (4.10) we have $\delta\left(\left(\Delta E^{B}+\Delta E^{A}\right) / \sqrt{a_{A} a_{B}}\right)$ multiplied, which always gives zero contribution when $\Delta E^{j}>0$. Then none of the above quantities $\mathcal{I}_{\varepsilon}^{W}$ or $\mathcal{I}_{\varepsilon}^{R}$ contribute to the non-zero expression of $\mathcal{I}_{\varepsilon}$. This signifies that for two observers accelerated parallelly, the condition for entanglement harvesting is not satisfied in $(1+3)$ dimensions.

In both $(1+1)$ and $(1+3)$ dimensions, considering parallelly accelerated detectors in a thermal bath, we observed that the condition for entanglement harvesting is not satisfied. This was also true in the case of accelerated detectors without a thermal bath, see $[4,38,40]$. Then one can deduce that here the thermal bath has no additional influence to make the entanglement harvesting possible. One should also notice that, it is not possible to distinguish between thermal and non-thermal scalar fields only by analyzing the parallelly accelerated detectors using entanglement harvesting information.

\subsection{Anti-parallel accelerations: harvesting possible}

In this subsection, we consider Alice in right and $B o b$ in the left Rindler wedge so that they are anti-parallelly accelerated in a thermal bath. To evaluate the integral $\mathcal{I}_{\varepsilon}$ and to reflect upon the previously obtained result by Reznik [4], where only the Wightman function rather than the Feynman propagator contributed to this integral, it is convenient to express it in the form of eq. (4.5). Here also we shall be separately evaluating the first integral $\mathcal{I}_{\varepsilon}^{W}$ and the second integral $\mathcal{I}_{\varepsilon}^{R}$, which respectively contains the Wightman function and the retarded Green's function, in $(1+1)$ and $(1+3)$ dimensions.

\subsection{1 (1+1) dimensions}

Analytical results. In $(1+1)$ dimensions the first part $\mathcal{I}_{\varepsilon}^{W}$ of the integral $\mathcal{I}_{\varepsilon}$, is estimated using the expression of the Wightman function from eq. (3.15). We have also considered $B o b$ (denoted by $B$ ) to be in LRW accelerating anti-parallelly to Alice in RRW (denoted by $A$ ). Then the integral $\mathcal{I}_{\varepsilon}^{W}$ becomes

$$
\begin{aligned}
& \mathcal{I}_{\varepsilon}^{W}=-\int_{-\infty}^{\infty} d \tau_{B} \int_{-\infty}^{\infty} d \tau_{A} e^{i\left(\Delta E^{B} \tau_{B}+\Delta E^{A} \tau_{A}\right)} G_{W_{L R}}^{\beta}\left(X_{B}, X_{A}\right) \\
& =-\delta\left(\frac{\Delta E^{B}-\Delta E^{A}}{\sqrt{a_{A} a_{B}}}\right) \frac{1}{\sqrt{\sinh \frac{\pi \Delta \widetilde{E}}{a_{A}} \sinh \frac{\pi \Delta \widetilde{E}}{a_{B}}}} \\
& \frac{\pi}{\Delta \widetilde{E} \sqrt{a_{A} a_{B}}}\left[\frac{e^{\frac{\pi \Delta \widetilde{E}}{2}\left(\frac{1}{a_{B}}-\frac{1}{a_{A}}\right)}}{1-e^{-\beta \Delta \widetilde{E}}}+\frac{e^{-\frac{\pi \Delta \widetilde{E}}{2}\left(\frac{1}{a_{B}}-\frac{1}{a_{A}}\right)}}{e^{\beta \Delta \widetilde{E}}-1}\right],
\end{aligned}
$$

where, $\Delta \widetilde{E}=\left(\Delta E^{B}+\Delta E^{A}\right) / 2$, and $\delta(z)$ denotes the Dirac delta distribution. For the evaluation of this integral we have made the change of variables $\tilde{v}=\tau_{B}+\tau_{A}$ and $\tilde{u}=\tau_{B}-\tau_{A}$. One may have considered moving to dimensionless variables $\gamma_{j}=\tau_{j} a_{j}$, and then make change of variables $\bar{v}=\gamma_{B}+\gamma_{A}$ and $\bar{u}=\gamma_{B}-\gamma_{A}$ to obtain the same final result. The Jacobian corresponding to both of these change of variables is $1 / 2$. Similarly one can evaluate the second integral $\mathcal{I}_{\varepsilon}^{R}$. Then using the Wightman function of eq. (2.8) the 
integral $\mathcal{I}_{\varepsilon}^{R}$ can be evaluated to be

$$
\begin{aligned}
\mathcal{I}_{\varepsilon}^{R} & =-\int_{-\infty}^{\infty} d \tau_{B} \int_{-\infty}^{\infty} d \tau_{A} \theta\left(\tau_{A}-\tau_{B}\right) e^{i\left(\Delta E^{B} \tau_{B}+\Delta E^{A} \tau_{A}\right)}\left[G_{W_{R L}}^{\beta}\left(X_{A}, X_{B}\right)-G_{W_{L R}}^{\beta}\left(X_{B}, X_{A}\right)\right] \\
& =\frac{\sinh \frac{\pi \Delta \widetilde{E}}{2}\left(\frac{1}{a_{B}}-\frac{1}{a_{A}}\right)}{2 \Delta \widetilde{E}} \frac{1}{\sqrt{\sinh \frac{\pi \Delta \widetilde{E}}{a_{A}} \sinh \frac{\pi \Delta \widetilde{E}}{a_{B}}}} \int_{-\infty}^{\infty} e^{-\frac{i}{2}\left(\Delta E^{B}-\Delta E^{A}\right) \tilde{u}} \theta(\tilde{u}) d \tilde{u},
\end{aligned}
$$

where, $\Delta \widetilde{E}=\left(\Delta E_{A}+\Delta E_{B}\right) / 2$. One can evaluate this last integral as

$$
\begin{aligned}
\int_{-\infty}^{\infty} e^{-\frac{i}{2}\left(\Delta E^{B}-\Delta E^{A}\right) \tilde{u}} \theta(\tilde{u}) d \tilde{u} & =\int_{0}^{\infty} e^{-\frac{i}{2}\left(\Delta E^{B}-\Delta E^{A}-i \epsilon\right) \tilde{u}} d \tilde{u} \\
& =\frac{2}{i\left(\Delta E^{B}-\Delta E^{A}-i \epsilon\right)}
\end{aligned}
$$

where a multiplicative regulator of $e^{-\epsilon \tilde{u} / 2}$, with $\epsilon>0$, is introduced in the integrand to evaluate this otherwise diverging integral. It is to be noted that the limit $\epsilon \rightarrow 0$ provides the actual value of the integral. One can express this quantity of eq. (4.14) with the help of a consequence of the Sokhotski-Plemelj theorem [60]

$$
\lim _{\epsilon \rightarrow 0+} \frac{1}{z-i \epsilon}=i \pi \delta(z)+\mathcal{P}\left(\frac{1}{z}\right)
$$

where, $\mathcal{P}(1 / z)$ denotes the principal value of $(1 / z)$, which is a finite quantity. Then in the limit of $\Delta E^{B} \rightarrow \Delta E^{A}$ the only contributing quantity in $\mathcal{I}_{\varepsilon}^{R}$ is

$$
\mathcal{I}_{\varepsilon}^{R}=\frac{\pi \sinh \left\{\frac{\pi \Delta \widetilde{E}}{2}\left(\frac{1}{a_{B}}-\frac{1}{a_{A}}\right)\right\}}{\Delta \widetilde{E} \sqrt{a_{A} a_{B}} \sqrt{\sinh \frac{\pi \Delta \widetilde{E}}{a_{A}} \sinh \frac{\pi \Delta \widetilde{E}}{a_{B}}}}\left[\delta\left(\frac{\Delta E^{B}-\Delta E^{A}}{\sqrt{a_{A} a_{B}}}\right)-\frac{i}{\pi} \mathcal{P}\left(\frac{\sqrt{a_{A} a_{B}}}{\Delta E^{B}-\Delta E^{A}}\right)\right] .
$$

The second quantity of the multiplicative term in the right hand side of this expression denotes the principle value and it is a finite quantity. It should be mentioned that when $\Delta E^{B} \neq \Delta E^{A}$ the Dirac delta distribution from eq. (4.16) vanishes and one is left out with only this finite second term. In this particular situation the integral $\mathcal{I}_{\varepsilon}^{W}$ also vanishes and the whole $\mathcal{I}_{\varepsilon}=\mathcal{I}_{\varepsilon}^{W}+\mathcal{I}_{\varepsilon}^{R}$ becomes finite. However, from eq. (4.1) it is observed that the integrals $\mathcal{I}_{j}$ still keeps the $\delta(0)$ terms in them. In that case it is obvious that the condition from (2.7) remains unfulfilled. On the other hand, when $\Delta E^{B}=\Delta E^{A}$ only the Dirac delta distribution contributes in the expression of $\mathcal{I}_{\varepsilon}^{R}$. In this situation, $\mathcal{I}_{\varepsilon}^{W}$ is also non zero, and there are multiplicative factors of $\delta(0)$ in $\mathcal{I}_{\varepsilon}^{W}$ and $\mathcal{I}_{\varepsilon}^{R}$ like the $\mathcal{I}_{j}$. Then it is evident that only for $\Delta E^{B}=\Delta E^{A}$ the condition (2.7) for entanglement harvesting may get satisfied.

Let us now make a comment on the contribution related to the retarded Green's function. It is observed from (4.16) that the integral $\mathcal{I}_{\varepsilon}^{R}$ in general vanishes when the two anti parallelly moving observers have equal magnitude of accelerations i.e. $a_{A}=a_{B}$. It is noticed that even in Green's function level when the accelerations of the anti-parallelly accelerated detectors are equal the quantity $G_{W_{R L}}^{\beta}\left(X_{A}, X_{B}\right)-G_{W_{L R}}^{\beta}\left(X_{B}, X_{A}\right)=0$, denoting the retarded Green's function in the integral of (4.13). This is expected as left Rindler wedge is causally disconnected from the right wedge. However, it remains non-zero for 
scenarios when $a_{A} \neq a_{B}$, although both are causally disconnected and retarded Green's function with respect to Minkowski mode vanishes (or when $a_{A}=0=a_{B}$ ) when they are spacelike separated. It may be mentioned that when $a_{A}=a_{B}$ then they can be regarded as "similar frames" (as LRW is mirror image of RRW) and hence since retarded Green's function vanishes in Minkowski frame, it must vanish in any other frame. Whereas for $a_{A} \neq a_{B}$ we do not have such similarity and we may take this as investigations of field operators from two "dis-similar frames". In this case the commutator of the fields may not be the same as it was earlier. In the above exactly this thing has been reflected in $\mathcal{I}_{\varepsilon}^{R}$. In summary, the relative acceleration between the frames introduces this non-triviality. We will see later that in $(1+3)$ case, compared to $(1+1)$ dimensional analysis, this has a big role to give a distinct feature in the entanglement harvesting.

From eq. (4.12) and (4.16) we obtain the expression of the integral $\mathcal{I}_{\varepsilon}$ corresponding to two anti-parallelly accelerated observers as $\mathcal{I}_{\varepsilon}=\mathcal{I}_{\varepsilon}^{W}+\mathcal{I}_{\varepsilon}^{R}$. As we have already discussed these expressions are non-zero and comparable to $\mathcal{I}_{j}$ only when $\Delta E^{A}$ and $\Delta E^{B}$ are equal, we then consider $\Delta E^{A}=\Delta E^{B}=\Delta E$. In that case we also have $\Delta \widetilde{E}=\Delta E$, and the condition for entanglement harvesting from eq. (2.7) becomes

$$
\begin{aligned}
& \left(\frac{e^{-\frac{\pi \Delta E}{a_{A}}}}{1-e^{-\beta \Delta E}}+\frac{e^{\frac{\pi \Delta E}{a_{A}}}}{e^{\beta \Delta E}-1}\right)\left(\frac{e^{-\frac{\pi \Delta E}{a_{B}}}}{1-e^{-\beta \Delta E}}+\frac{e^{\frac{\pi \Delta E}{a_{B}}}}{e^{\beta \Delta E}-1}\right)< \\
& 4\left[\frac{e^{\frac{\pi \Delta E}{2}}\left(\frac{1}{a_{B}}-\frac{1}{a_{A}}\right)}{1-e^{-\beta \Delta E}}+\frac{e^{-\frac{\pi \Delta E}{2}\left(\frac{1}{a_{B}}-\frac{1}{a_{A}}\right)}}{e^{\beta \Delta E}-1}-\sinh \left\{\frac{\pi \Delta E}{2}\left(\frac{1}{a_{B}}-\frac{1}{a_{A}}\right)\right\}\right]^{2} .
\end{aligned}
$$

From this expression (4.17) depicting the condition for entanglement harvesting for two anti-parallelly accelerated observers, we see that contribution of the retarded Green's function exists when the detectors have different magnitudes of acceleration, i.e., $a_{A} \neq a_{B}$.

Numerical analysis. In figure 1 we have plotted the quantity $\mathcal{C}_{\mathcal{I}}$ of $(2.10)$ signifying the concurrence, with respect to $\sigma$, which is proportional to the inverse temperature of the thermal bath $\sigma=\beta \Delta E$, considering two anti-parallelly accelerated observers with different accelerations in $(1+1)$ dimensions. The curves in this figure correspond to fixed $\alpha_{B}=1$ and different fixed $\alpha_{A}$. It is to be noted that the quantity $\mathcal{C}_{\mathcal{I}}$ plotted in the figure is obtained using the expressions from (4.1), (4.12) and (4.16) when $\Delta E^{B}=\Delta E^{A}$ without the multiplicative $\delta(0)$ term. Removing this delta function from $\mathcal{C}_{\mathcal{I}}$ can be interpreted as taking a rate per unit proper time, like discussed in literature [40]. From figure 1 we have the following observations.

- For low acceleration $\alpha_{A}$ of the first detector (e.g. $\alpha_{A}=1 / 2$ ) the quantity $\mathcal{C}_{\mathcal{I}}$ is negative for very high temperature of the thermal bath, and it tends to increase with increasing $\beta$ and becomes positive at some much large $\beta$ or low temperature of the thermal bath. Therefore thermal fields do not allow entanglement at high temperature. Entanglement can start only from certain value of temperature of thermal bath to lower values when the first detector moves with small acceleration. 


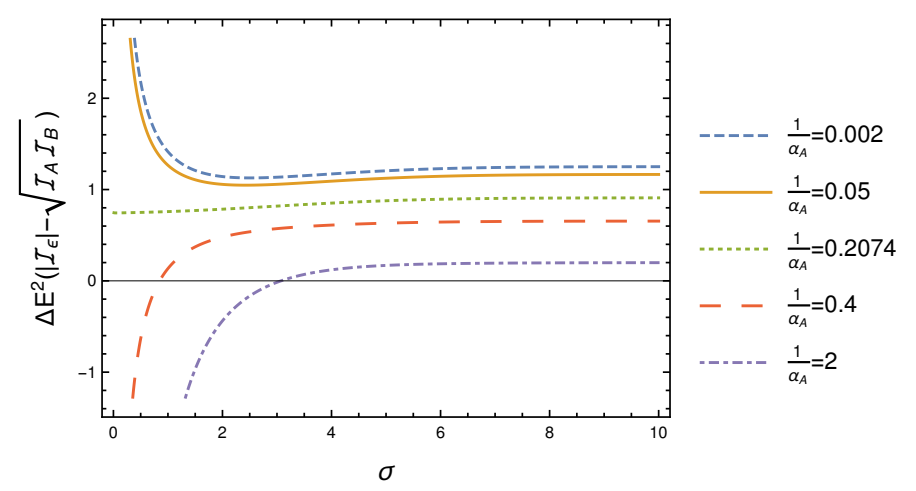

Figure 1. In $(1+1)$ dimensions the quantity $\Delta E^{2}\left(\left|\mathcal{I}_{\varepsilon}\right|-\sqrt{\mathcal{I}_{A} \mathcal{I}_{B}}\right)$ is plotted for two anti-parallelly accelerating detectors with respect to the inverse temperature $\sigma=\beta \Delta E$ for different fixed $\alpha_{A}=$ $a_{A} / \Delta E$. The other parameter is fixed at $\alpha_{B}=a_{B} / \Delta E=1$.

- For high acceleration $\alpha_{A}$ of the first detector $\left(\alpha_{A}=1 / 0.002\right)$ the quantity $\mathcal{C}_{\mathcal{I}}$ is positive for very high temperature of the thermal bath, and it tends to decrease with increasing $\beta$ but never becomes negative at much larger $\beta$ or low temperature of the thermal bath. So for large values of acceleration, we will have entanglement at any temperature of bath.

- With these it is observed that there is a characteristic change in the nature of these curves depending on the value of $\alpha_{A}$ - for low values of $\alpha_{A}$ the entanglement increases with increase of $\beta$ while after certain value of $\alpha_{A}$ entanglement decreases with increase of $\beta$. We call the value of acceleration $\alpha_{A}=\alpha_{c}$ as critical value around which these curves have different nature. In figure 1 this is given by the almost straight line which is green in color.

To find this critical value note that the change is nature is prominent for very low value of $\beta$; i.e. at higher temperature of the bath. So it will be sufficient to investigate $\mathcal{C}_{\mathcal{I}}$ for very low value of $\sigma$. Also in this regime the critical line (which is green in figure 1) is straight. Therefore the vanishing of the slope of the curve in low value of $\sigma$ will yield $\alpha_{c}$. One can series expand the derivative of $\mathcal{C}_{\mathcal{I}}$ with respect to $\sigma$ in this case, in small $\sigma$ regimes and observe that it is of the form $(\partial / \partial \sigma) \mathcal{C}\left(\rho_{A B}\right)=\left(1 / \sigma^{2}\right) \mathcal{C}_{1}+\mathcal{C}_{2}+\mathcal{O}(\sigma)$. Then in high temperature regime one can predict about the transition point $\alpha_{c}$ by making $\mathcal{C}_{1}=0$ (this is leading term) with $\alpha_{A}=\alpha_{c}$, which provides us with the expression

$$
\mathcal{C}_{1}=\frac{\pi}{\sqrt{\sinh \left(\frac{\pi}{\alpha_{c}}\right) \sinh \left(\frac{\pi}{\alpha_{B}}\right)}}\left(\sqrt{\cosh \left(\frac{\pi}{\alpha_{c}}\right) \cosh \left(\frac{\pi}{\alpha_{B}}\right)}-2 \cosh \left(\frac{\pi\left(\alpha_{B}-\alpha_{c}\right)}{2 \alpha_{c} \alpha_{B}}\right)\right)=0 .
$$

It can be checked that the above equation yields the value of $\alpha_{c}$ as 4.82026 for our choice of parameter value $\alpha_{B}=1$. Note that this is exactly the value of $\alpha_{A}$ for which the critical curve (green in color in figure 1) was obtained numerically. 


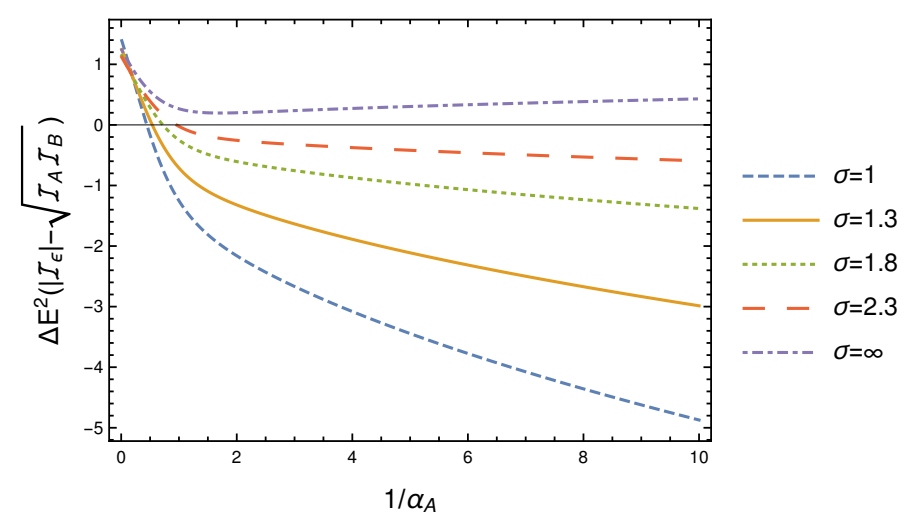

Figure 2. In $(1+1)$ dimensions the quantity $\Delta E^{2}\left(\left|\mathcal{I}_{\varepsilon}\right|-\sqrt{\mathcal{I}_{A} \mathcal{I}_{B}}\right)$ is plotted for two anti-parallelly accelerating detectors with respect to the acceleration of the first detector $\alpha_{A}$ for different fixed inverse temperature of the thermal bath $\sigma$. The other parameters are fixed at $\alpha_{B}=1$.

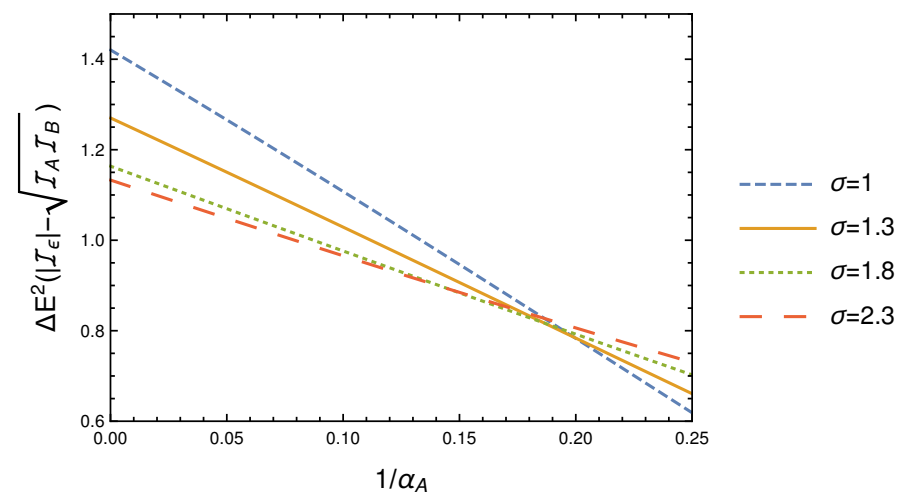

Figure 3. In $(1+1)$ dimensions the quantity $\Delta E^{2}\left(\left|\mathcal{I}_{\varepsilon}\right|-\sqrt{\mathcal{I}_{A} \mathcal{I}_{B}}\right)$ is plotted for two anti-parallelly accelerating detectors with respect to the acceleration of the first detector $\alpha_{A}$ for different fixed inverse temperatures of the thermal bath $\sigma$. The other parameters are fixed at $\alpha_{B}=1$. In particular we have depicted the curves of figure 2 in lower regime of $1 / \alpha_{A}$.

In figure 2 we have plotted $\mathcal{C}_{\mathcal{I}}$ signifying the concurrence with respect to the acceleration of the first detector $\alpha_{A}$ for different fixed $\sigma$. From this figure one can observe that the temperature of the thermal bath has a diminishing effect on the entanglement measure for low values of the acceleration of the first detector $\alpha_{A}$. It is observed that for smaller $\alpha_{A}$ with low $\beta$ (if $\Delta E$ is kept fixed then $\beta$ changes in unison with $\sigma$ ), i.e., for very high temperature, the condition for entanglement harvesting is failing, while for high $\alpha_{A}$ the condition again gets satisfied. We have also depicted the same curves as shown in figure 2 in lower regimes of $1 / \alpha_{A}$ in figure 3. From this curve we observe that, above a certain value of $\alpha_{A}$, which is the critical value $\alpha_{c}$, the thermal bath has an enhancing effect on concurrence (denoted by $\mathcal{C}_{\mathcal{I}}$ ). Then the plots depicted in figure 2 and 3 together predict the same phenomena provided by figure 1, i.e., for low accelerations thermal bath has a diminishing effect and for high accelerations thermal bath has an enhancing effect on the entanglement measure, and there is a perceivable critical value of acceleration separating 


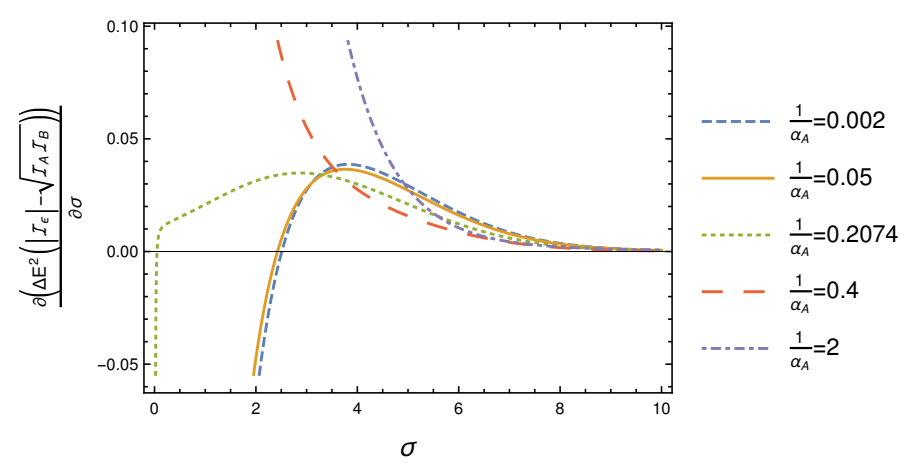

Figure 4. In $(1+1)$ dimensions the derivative with respect to $\sigma$ of the quantity $\Delta E^{2}\left(\left|\mathcal{I}_{\varepsilon}\right|-\sqrt{\mathcal{I}_{A} \mathcal{I}_{B}}\right)$ is plotted for two anti-parallelly accelerating detectors for varying $\sigma$. The other parameters $\alpha_{B}=1$ and $\alpha_{A}$ are fixed.

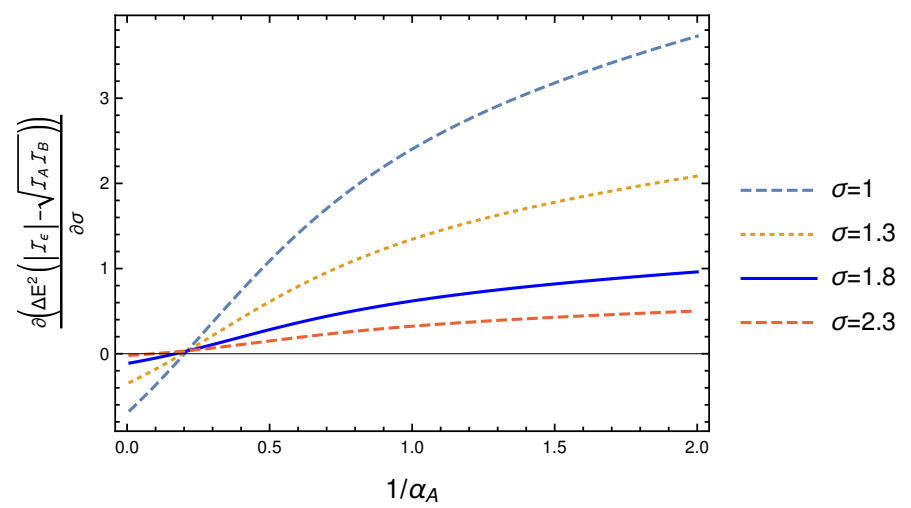

Figure 5. In $(1+1)$ dimensions the derivative with respect to $\sigma$ of the quantity $\Delta E^{2}\left(\left|\mathcal{I}_{\varepsilon}\right|-\sqrt{\mathcal{I}_{A} \mathcal{I}_{B}}\right)$ is plotted for two anti-parallelly accelerating detectors for varying acceleration of the first detector $\alpha_{A}$. The temperatures of the thermal bath $\sigma$ and other parameter $\alpha_{B}=1$ are fixed.

these two regimes of accelerations. In figure 4 and 5 we have further plotted the derivative of $\mathcal{C}_{\mathcal{I}}$ with respect to $\sigma$ for varying $\sigma$ and $\alpha_{A}$ for the perception of $\alpha_{c}$. Figure 4 shows that some curves contains negative slope while others have positive slope for initial values of $\beta$. Similarly figure 5 signifies that the derivative of the quantity, denoting concurrence, with respect to $\sigma$ becomes zero at a particular value of $\alpha_{A}$. All these reassured the existence the aforesaid critical value of $\alpha_{A}$.

It is to be noted that in the equal magnitude of acceleration limit the second integral from eq. (4.16) coming from the retarded Green's function vanishes and one is left with only $\mathcal{I}_{\varepsilon}=\mathcal{I}_{\varepsilon}^{W}$. In this particular case we consider $a_{A}=a_{B}=a$, and the condition for entanglement harvesting from eq. (2.7) is then given by

$$
\frac{e^{-\frac{\pi \Delta E}{a}}}{1-e^{-\beta \Delta E}}+\frac{e^{\frac{\pi \Delta E}{a}}}{e^{\beta \Delta E}-1}<2 \frac{e^{\beta \Delta E}+1}{e^{\beta \Delta E}-1},
$$

which for the zero temperature of the thermal bath, i.e., in the $\beta \rightarrow \infty$ limit, becomes $e^{\frac{\pi \Delta E}{a}}>1 / 2$. This basically reinstates the fact that in the zero temperature case the entanglement can be harvested for anti-parallelly accelerated detectors with any possible equal 


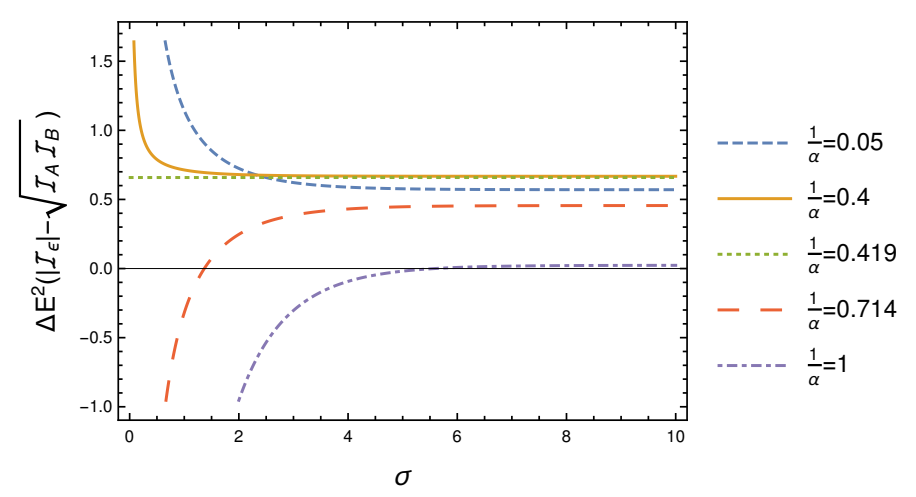

Figure 6. In $(1+1)$ dimensions the quantity $\Delta E^{2}\left(\left|\mathcal{I}_{\varepsilon}\right|-\sqrt{\mathcal{I}_{A} \mathcal{I}_{B}}\right)$ is plotted for two anti-parallelly accelerating detectors with respect to the inverse temperature of the thermal bath $\sigma$ for equal magnitude of proper accelerations, i.e., $\alpha_{A}=\alpha_{B}$.

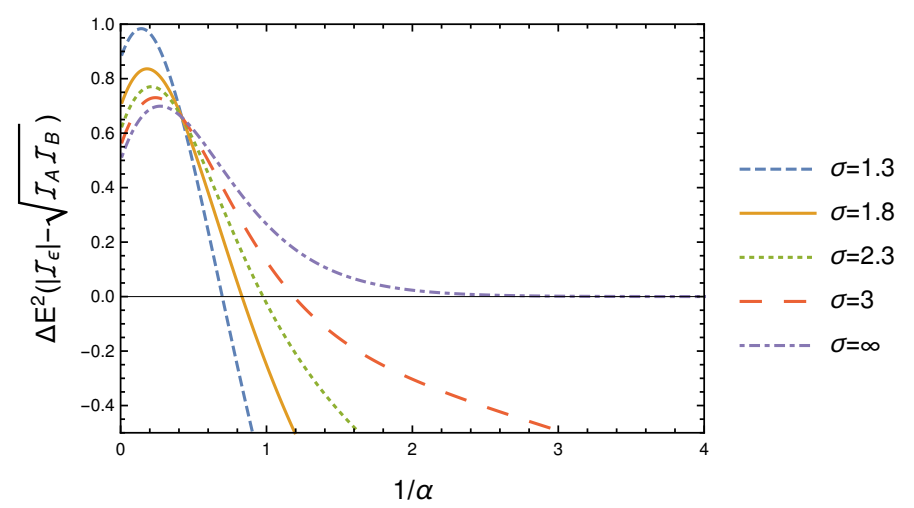

Figure 7. In $(1+1)$ dimensions the quantity $\Delta E^{2}\left(\left|\mathcal{I}_{\varepsilon}\right|-\sqrt{\mathcal{I}_{A} \mathcal{I}_{B}}\right)$ is plotted for two anti-parallelly accelerating detectors with same magnitude of acceleration for varying acceleration of the detectors $\alpha$ and different fixed $\sigma$.

acceleration, which is also observed from $[4,40]$ though considering the detectors in $(1+3)$ dimensions. In figure 6 and figure 7 we have plotted $\mathcal{C}_{\mathcal{I}}=\left|\mathcal{I}_{\varepsilon}\right|-\mathcal{I}_{j}$ respectively with respect to varying $\sigma$ and $\alpha$. From these figures also we observe the same phenomena as perceived before. Here also we see that below a certain critical $\alpha$ entanglement harvesting is not possible for low $\beta$ or high temperature of the thermal bath and the entanglement measure increases with increasing $\beta$. On the other hand, above this critical acceleration entanglement measure decreases with increasing $\beta$, but remains positive. In figure 7 this behavioral change of the curves after a certain critical acceleration $\alpha_{c}$ is much more prominent than the previous ones with different accelerations. It should be noted that in this equal acceleration case, by making the derivative of the quantity $\mathcal{C}_{\mathcal{I}}$ with respect to $\sigma$ equal to zero, one can obtain the critical value of acceleration $\alpha_{c}=\pi / \log [2+\sqrt{3}]$, which is around $\alpha_{c} \approx 2.385$ and is independent of $\sigma$. This is depicted by a straight line in figure 6 . In figure 8 and 9 the derivative of $\mathcal{C}_{\mathcal{I}}$ is plotted with respect to varying $\alpha$ and $\sigma$, which also signifies the earlier mentioned slope change about the critical value of $\alpha$. This reconfirms the existence of the aforesaid criticality. 


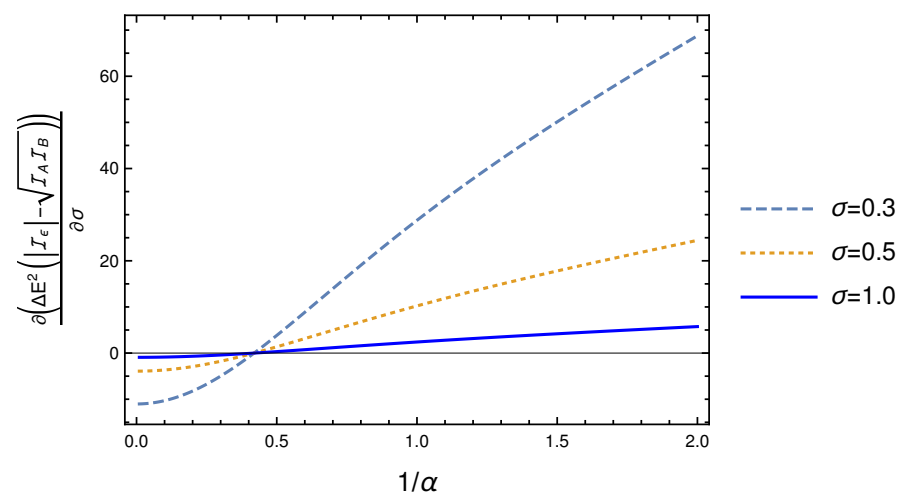

Figure 8. In $(1+1)$ dimensions the quantity $(\partial / \partial \sigma)\left(\Delta E^{2}\left(\left|\mathcal{I}_{\varepsilon}\right|-\sqrt{\mathcal{I}_{A} \mathcal{I}_{B}}\right)\right)$ is plotted for two anti-parallelly accelerating detectors with respect to the equal magnitude of proper accelerations $\alpha_{A}=\alpha_{B}$ for fixed inverse temperature of the thermal bath $\sigma$. The critical acceleration, where this quantity is $\sigma$ independent, is $\alpha_{c}=2.3854$.

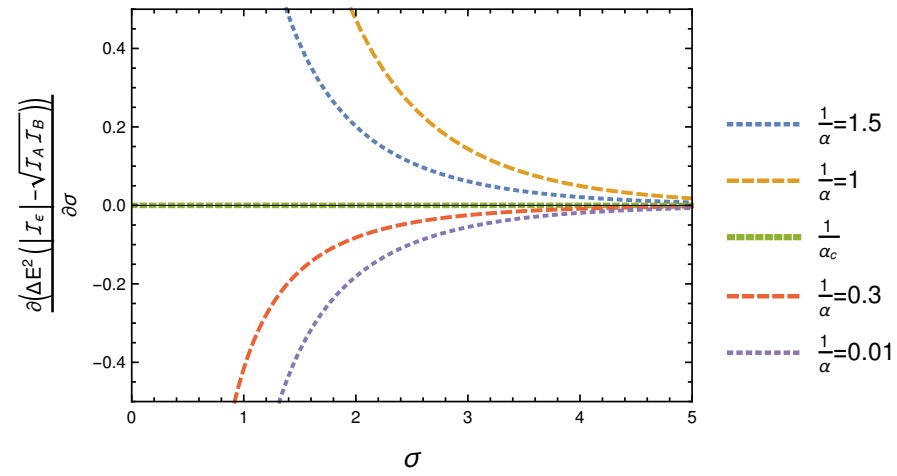

Figure 9. In $(1+1)$ dimensions the quantity $(\partial / \partial \sigma)\left(\Delta E^{2}\left(\left|\mathcal{I}_{\varepsilon}\right|-\sqrt{\mathcal{I}_{A} \mathcal{I}_{B}}\right)\right)$ is plotted for two anti-parallelly accelerating detectors with respect to the inverse temperature of the thermal bath $\sigma$ for equal magnitude of proper accelerations $\alpha_{A}=\alpha_{B}$. The critical acceleration, where this quantity is $\sigma$ independent, is $\alpha_{c}=2.3854$.

\subsection{2 (1+3) dimensions}

Analytical results. From eq. (2.8) we observe that there are two specific terms in the integral $\mathcal{I}_{\varepsilon}$. One involving a Wightman function and another involving a retarded Green's function. The second term involving the retarded Green's function is recently conceived through rigorous analysis of the model for entanglement harvesting. Like the $(1+1)$ dimensional case in $(1+3)$ dimensions also we shall explicitly evaluate these terms. We express the first quantity using the Wightman function of eq. (3.17), i.e., considering the observer $B$ to be accelerating anti-parallelly in LRW with respect to observer $A$ in RRW, as

$$
\begin{aligned}
\mathcal{I}_{\varepsilon}^{W} & =-\int_{-\infty}^{\infty} d \tau_{B} \int_{-\infty}^{\infty} d \tau_{A} e^{i\left(\Delta E^{B} \tau_{B}+\Delta E^{A} \tau_{A}\right)} G_{W_{L R}^{3 D}}^{\beta}\left(X_{B}, X_{A}\right) \\
& =-\delta\left(\frac{\Delta E^{B}-\Delta E^{A}}{\sqrt{a_{A} a_{B}}}\right) \frac{1}{a_{A} a_{B}} \frac{\Upsilon\left(\Delta \widetilde{E}, a_{B}, a_{A}\right)}{\pi}\left[\frac{e^{\frac{\pi \Delta \widetilde{E}}{2}\left(\frac{1}{a_{B}}-\frac{1}{a_{A}}\right)}}{1-e^{-\beta \Delta \widetilde{E}}}+\frac{e^{-\frac{\pi \Delta \widetilde{E}}{2}\left(\frac{1}{a_{B}}-\frac{1}{a_{A}}\right)}}{e^{\beta \Delta \widetilde{E}}-1}\right]
\end{aligned}
$$


where, $\Delta \widetilde{E}=\left(\Delta E^{B}+\Delta E^{A}\right) / 2$, and $\delta(z)$ denotes the Dirac delta distribution. For the evaluation of this integral we have considered change of variables $\tilde{v}=\tau_{B}+\tau_{A}$ and $\tilde{u}=$ $\tau_{B}-\tau_{A}$, and we shall be using this same change of variables to evaluate the next integral also. Then one can evaluate the second part of the integral $\mathcal{I}_{\varepsilon}$ from eq. $(2.8)$ as

$$
\begin{aligned}
\mathcal{I}_{\varepsilon}^{R} & =-\int_{-\infty}^{\infty} d \tau_{B} \int_{-\infty}^{\infty} d \tau_{A} \theta\left(\tau_{A}-\tau_{B}\right) e^{i\left(\Delta E^{B} \tau_{B}+\Delta E^{A} \tau_{A}\right)}\left[G_{W_{R L}^{3 D}}^{\beta}\left(X_{A}, X_{B}\right)-G_{W_{L R}^{3 D}}^{\beta}\left(X_{B}, X_{A}\right)\right] \\
& =\sinh \left\{\frac{\pi \Delta \widetilde{E}}{2}\left(\frac{1}{a_{B}}-\frac{1}{a_{A}}\right)\right\} \frac{2 \Upsilon\left(\Delta \widetilde{E}, a_{B}, a_{A}\right)}{(2 \pi)^{2} \sqrt{a_{A} a_{B}}} \int_{-\infty}^{\infty} e^{-\frac{i}{2}\left(\Delta E^{B}-\Delta E^{A}\right) \tilde{u}} \theta(\tilde{u}) d \tilde{u}
\end{aligned}
$$

Like the previous $(1+1)$ dimensional case, using (4.15) the contributing part of this expression here can be evaluated to be

$$
\begin{aligned}
\mathcal{I}_{\varepsilon}^{R}= & \frac{\sinh \left\{\frac{\pi \Delta \widetilde{E}}{2}\left(\frac{1}{a_{B}}-\frac{1}{a_{A}}\right)\right\} \Upsilon\left(\Delta \widetilde{E}, a_{B}, a_{A}\right)}{\pi a_{A} a_{B}} \\
& \times\left[\delta\left(\frac{\Delta E^{B}-\Delta E^{A}}{\sqrt{a_{A} a_{B}}}\right)-\frac{i}{\pi} \mathcal{P}\left(\frac{\sqrt{a_{A} a_{B}}}{\Delta E^{B}-\Delta E^{A}}\right)\right] .
\end{aligned}
$$

Like the $(1+1)$ dimensional case here also we observe that when $\Delta E^{B} \neq \Delta E^{A}$ the integral $\mathcal{I}_{\varepsilon}^{R}$ becomes finite, whereas $\mathcal{I}_{\varepsilon}^{W}$ vanishes. On the other hand, from (4.2) we observe that the integrals $\mathcal{I}_{j}$ have a multiplicative $\delta(0)$ term in them. Then in this situation one cannot harvest any entanglement. Entanglement harvesting may become possible only when $\Delta E^{B}=\Delta E^{A}$. In that case we consider $\Delta E^{B}=\Delta E^{A}=\Delta E$, which also results in $\Delta \widetilde{E}=\Delta E$. Then from eq. (4.20) and (4.22) one can obtain the expression of the integral $\mathcal{I}_{\varepsilon}$ corresponding to two anti-parallelly accelerated observers in $(1+3)$ dimensions as $\mathcal{I}_{\varepsilon}=\mathcal{I}_{\varepsilon}^{W}+\mathcal{I}_{\varepsilon}^{R}$, and then get the condition for entanglement harvesting (2.7) to be

$$
\begin{aligned}
& \left(\frac{e^{-\frac{\pi \Delta E}{a_{A}}}}{1-e^{-\beta \Delta E}}+\frac{e^{\frac{\pi \Delta E}{a_{A}}}}{e^{\beta \Delta E}-1}\right)\left(\frac{e^{-\frac{\pi \Delta E}{a_{B}}}}{1-e^{-\beta \Delta E}}+\frac{e^{\frac{\pi \Delta E}{a_{B}}}}{e^{\beta \Delta E}-1}\right) \Upsilon\left(\Delta E, a_{A}, a_{A}\right) \Upsilon\left(\Delta E, a_{B}, a_{B}\right)< \\
& 4\left[\frac{e^{\frac{\pi \Delta E}{2}}\left(\frac{1}{a_{B}}-\frac{1}{a_{A}}\right)}{1-e^{-\beta \Delta E}}+\frac{e^{-\frac{\pi \Delta E}{2}\left(\frac{1}{a_{B}}-\frac{1}{a_{A}}\right)}}{e^{\beta \Delta E}-1}-\sinh \left\{\frac{\pi \Delta E}{2}\left(\frac{1}{a_{B}}-\frac{1}{a_{A}}\right)\right\}\right]^{2} \Upsilon\left(\Delta E, a_{A}, a_{B}\right)^{2} .
\end{aligned}
$$

Numerical analysis. In figure 10 we have plotted the quantity $\mathcal{C}_{\mathcal{I}}=\Delta E^{2}\left(\left|\mathcal{I}_{\varepsilon}\right|-\sqrt{\mathcal{I}_{A} \mathcal{I}_{B}}\right)$, which signifies the concurrence, with respect to $\alpha_{A}$ for different fixed temperature of the thermal bath. Like the $(1+1)$ dimensional case here also we have removed the $\delta(0)$ factor from $\mathcal{C}_{\mathcal{I}}$, which now describes a rate of concurrence per unit proper time. On the other hand, in figure 11 and figure 12 we have plotted this $\mathcal{C}_{\mathcal{I}}$ with respect to the inverse temperature of the thermal bath $\sigma=\beta \Delta E$ for different fixed $\alpha_{A}$. From both of these figures we observe that higher temperature of the thermal bath results in a failure of the condition for entanglement harvesting for accelerations much lower than the critical acceleration, which is in agreement with the understandings gained from the $(1+1)$ dimensional analysis. However, the characteristics of the curves obtained from figure 10 are turbulent compared 


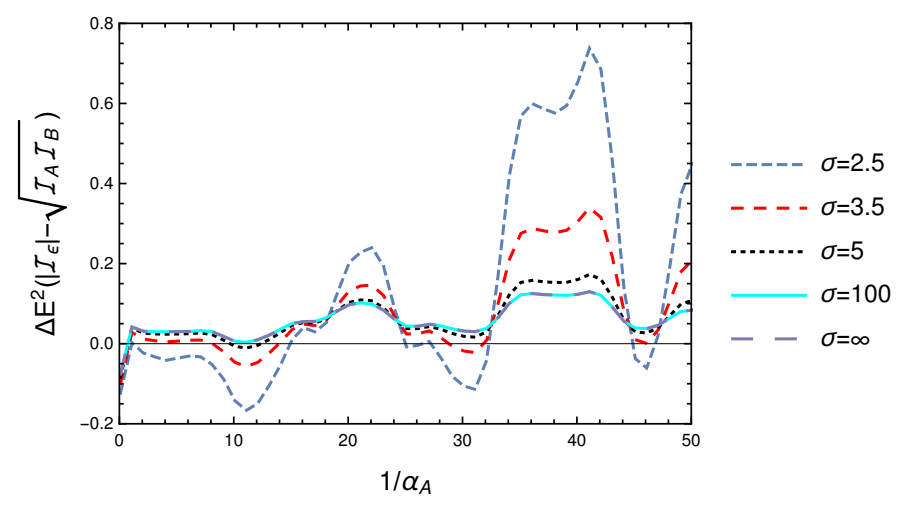

Figure 10. In $(1+3)$ dimensions the quantity $\Delta E^{2}\left(\left|\mathcal{I}_{\varepsilon}\right|-\sqrt{\mathcal{I}_{A} \mathcal{I}_{B}}\right)$ is plotted for two anti-parallelly accelerating detectors with respect to the acceleration of the first detector $\alpha_{A}$ for different fixed $\sigma$. The other parameter is fixed at $\alpha_{B}=1$.

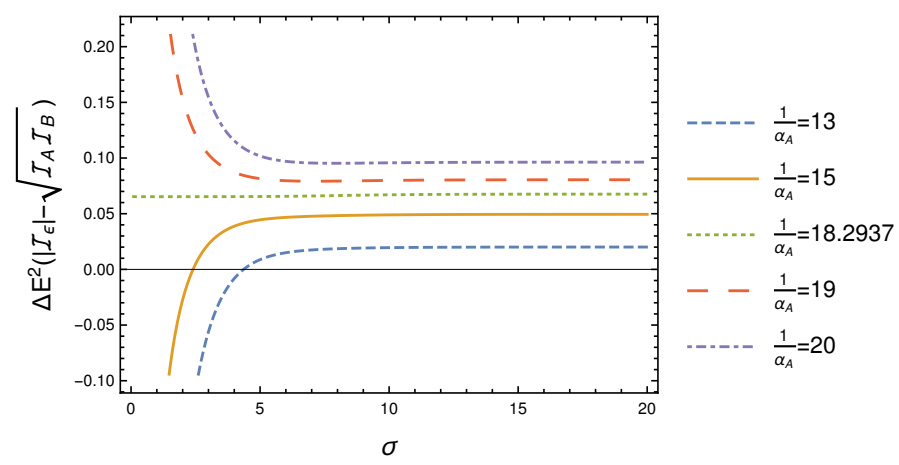

Figure 11. In $(1+3)$ dimensions the quantity $\Delta E^{2}\left(\left|\mathcal{I}_{\varepsilon}\right|-\sqrt{\mathcal{I}_{A} \mathcal{I}_{B}}\right)$ is plotted for two anti-parallelly accelerating detectors with respect to the inverse temperature of the thermal bath $\sigma$ for different fixed accelerations $\alpha_{A}$. The other parameter is fixed at $\alpha_{B}=1$.

to the $(1+1)$ dimensional curves of figure 2 in similar situation. It is also noticed that unlike the $(1+1)$ dimensional case there are multiple transition points of $\alpha_{A}$ in curves of figure 10. After crossing each of these transition points the characteristics of $\mathcal{C}_{\mathcal{I}}$ flips with respect to $\beta$, i.e., in some of the regions, in between these transition points, $\mathcal{C}_{\mathcal{I}}$ increases with increasing $\beta$, and in the neighboring regions $\mathcal{C}_{\mathcal{I}}$ decreases with increasing $\beta$. In figure 13 and 14 we have plotted the derivative of $\mathcal{C}_{\mathcal{I}}$ with respect to $\sigma$ for varying $\sigma$ and $\alpha_{A}$ to further confirm the positions of the transition points. Another intriguing thing to notice is that in $(1+1)$ dimensions for $\alpha_{A} \neq \alpha_{B}$ we observed that for a fixed temperature of the thermal field entanglement harvesting is possible for any accelerations above a certain acceleration. However, here in $(1+3)$ dimensions this is not the case, as now entanglement harvesting is possible in discrete ranges of $\alpha_{A}$ for certain values of fixed temperatures of the thermal fields.

It is to be noted that in the equal magnitude of acceleration limit the second integral from eq. (4.22) coming from the retarded Green's function vanishes and one is left with only $\mathcal{I}_{\varepsilon}=\mathcal{I}_{\varepsilon}^{W}$. In this particular case $a_{A}=a_{B}=a$, and the condition for entanglement 


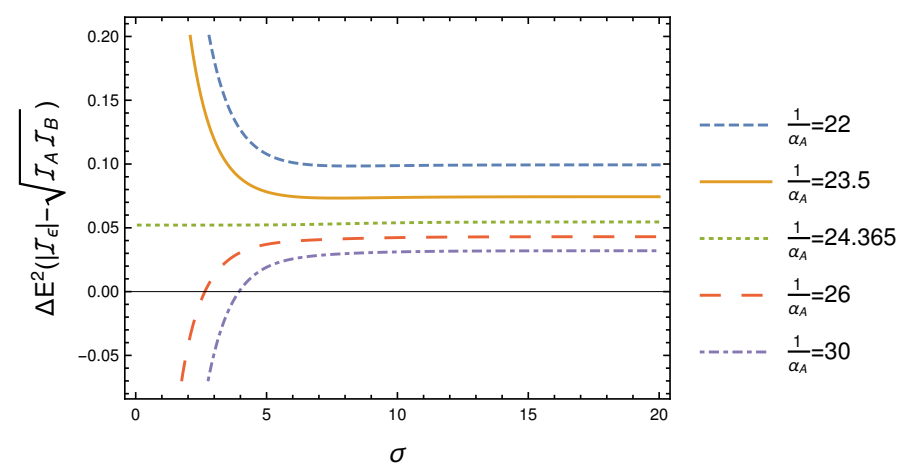

Figure 12. In $(1+3)$ dimensions the quantity $\Delta E^{2}\left(\left|\mathcal{I}_{\varepsilon}\right|-\sqrt{\mathcal{I}_{A} \mathcal{I}_{B}}\right)$ is plotted for two anti-parallelly accelerating detectors with respect to the inverse temperature of the thermal bath $\sigma$ for different fixed accelerations $\alpha_{A}$, and $\alpha_{B}$ is fixed at $\alpha_{B}=1$. Here the set of fixed $\alpha_{A}$ is different than the ones considered in figure 11. However, here also one can observe a transition in the nature of the curves as $\alpha_{A}$ changes.

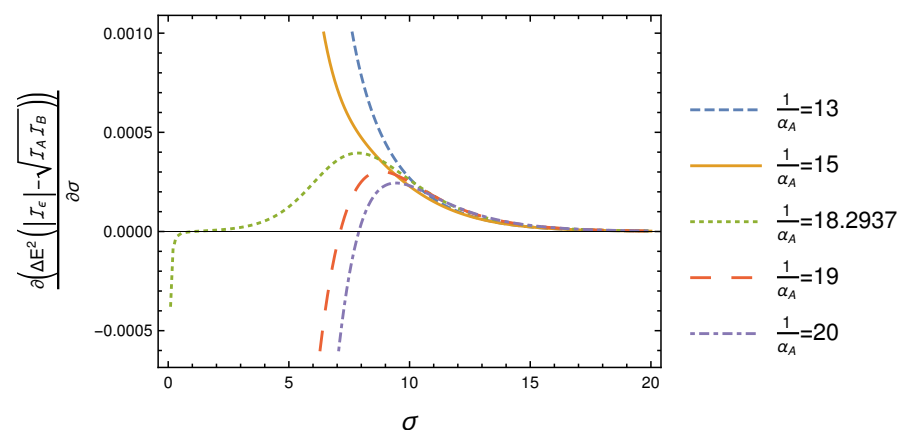

Figure 13. In $(1+3)$ dimensions the derivative with respect to $\sigma$ of the quantity $\Delta E^{2}\left(\left|\mathcal{I}_{\varepsilon}\right|-\sqrt{\mathcal{I}_{A} \mathcal{I}_{B}}\right)$ is plotted for two anti-parallelly accelerating detectors for varying $\sigma$. The other parameters $\alpha_{B}=1$ and $\alpha_{A}$ are fixed.

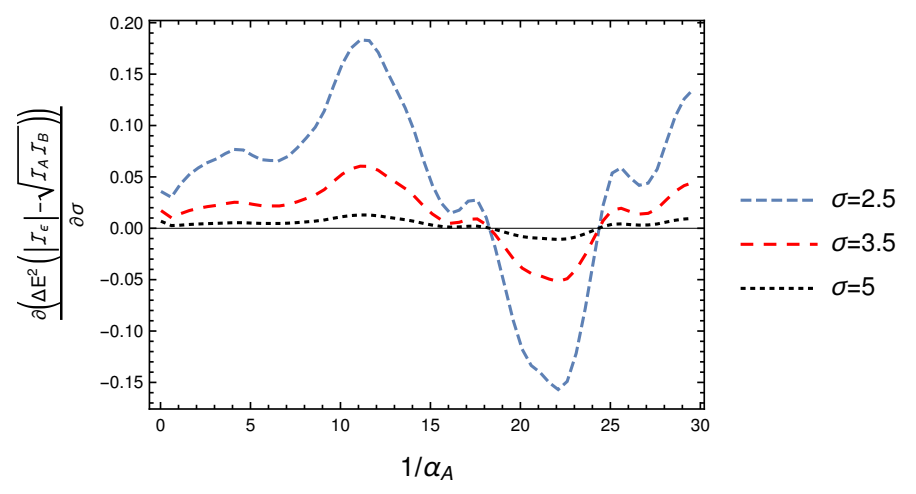

Figure 14. In $(1+3)$ dimensions the derivative with respect to $\sigma$ of the quantity $\Delta E^{2}\left(\left|\mathcal{I}_{\varepsilon}\right|-\sqrt{\mathcal{I}_{A} \mathcal{I}_{B}}\right)$ is plotted for two anti-parallelly accelerating detectors for varying acceleration of the first detector $\alpha_{A}$. The temperatures of the thermal bath $\sigma$ and other parameter $\alpha_{B}=1$ are fixed. 


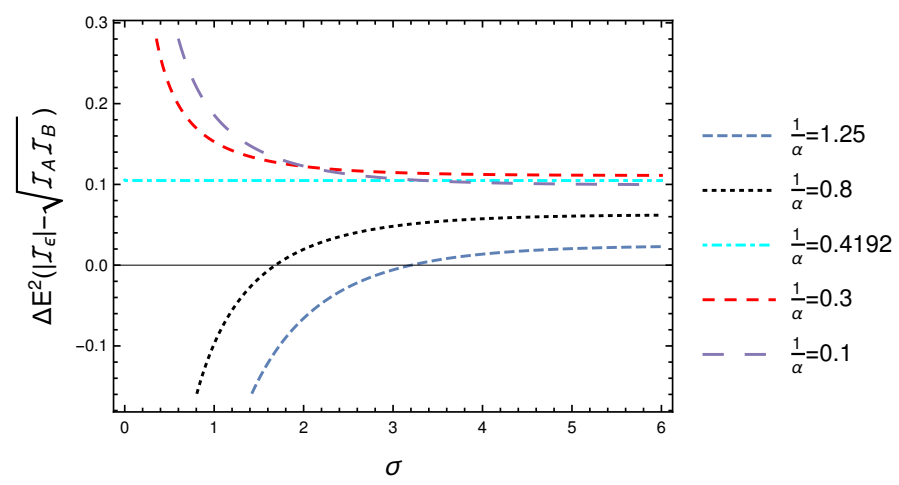

Figure 15. In $(1+3)$ dimensions the quantity $\Delta E^{2}\left(\left|\mathcal{I}_{\varepsilon}\right|-\sqrt{\mathcal{I}_{A} \mathcal{I}_{B}}\right)$ is plotted for two anti-parallelly accelerating detectors with respect to the inverse temperature of the thermal bath $\sigma$ for equal magnitude of proper accelerations, i.e., $\alpha_{A}=\alpha_{B}$.

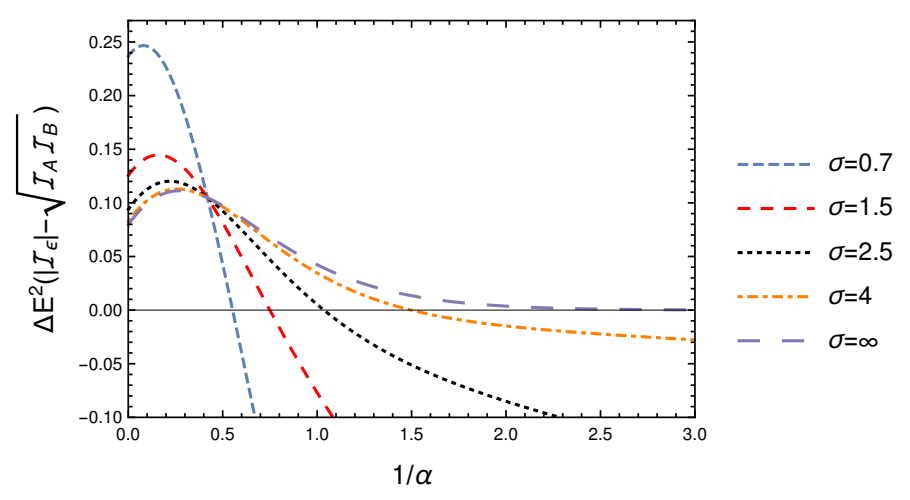

Figure 16. In $(1+3)$ dimensions the quantity $\Delta E^{2}\left(\left|\mathcal{I}_{\varepsilon}\right|-\sqrt{\mathcal{I}_{A} \mathcal{I}_{B}}\right)$ is plotted for two anti-parallelly accelerating detectors with respect to $1 / \alpha$ for fixed inverse temperatures of the thermal bath $\sigma$ for equal magnitude of proper accelerations, i.e., $\alpha_{A}=\alpha_{B}$.

harvesting from eq. (4.23) becomes same as the one from the $(1+1)$ dimensional case of eq. (4.19). Then it is expected that the entanglement measure $\mathcal{C}_{\mathcal{I}}$ in $(1+3)$ should be qualitatively same as the one from $(1+1)$ dimensions. However, it is quantitatively different in the $(1+3)$ dimensional case compared to the $(1+1)$ dimensional case with equal acceleration. In figure 15 and 16 we have further plotted this quantity $\mathcal{C}_{\mathcal{I}}$ signifying the concurrence, in this case in $(1+3)$ dimensions. Here also the concurrence shows similar characteristics as was observed in the $(1+1)$ dimensional case. From figure 16 it is clear that the temperature of the thermal bath diminishes the range of acceleration in which entanglement extraction is possible. However, it enhances the amount of concurrence above a certain value of acceleration thus enhancing the entanglement extraction in that region. Furthermore, in figure 17 and 18 we have plotted the derivative of $\mathcal{C}_{\mathcal{I}}$ with respect to $\sigma$ in this case for varying $\sigma$ and $\alpha$ for the perception of $\alpha_{c}$. It should be noted that in $(1+3)$ dimensions one is left out with only one transition point, contrary to multiple transition points in $\alpha_{A}$ from figure 10, when equal accelerations are considered. 


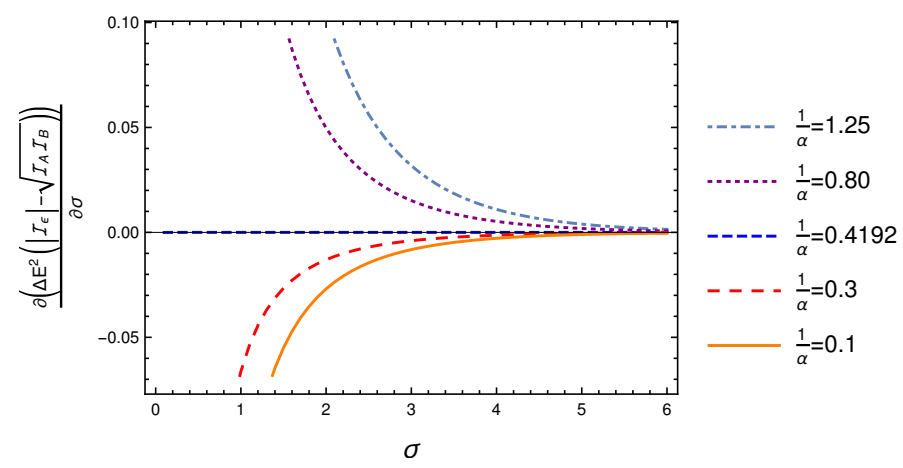

Figure 17. In $(1+3)$ dimensions the quantity $(\partial / \partial \sigma)\left(\Delta E^{2}\left(\left|\mathcal{I}_{\varepsilon}\right|-\sqrt{\mathcal{I}_{A} \mathcal{I}_{B}}\right)\right)$ is plotted for two anti-parallelly accelerating detectors with respect to the inverse temperature of the thermal bath $\sigma$ for equal magnitude of proper accelerations $\alpha_{A}=\alpha_{B}$.

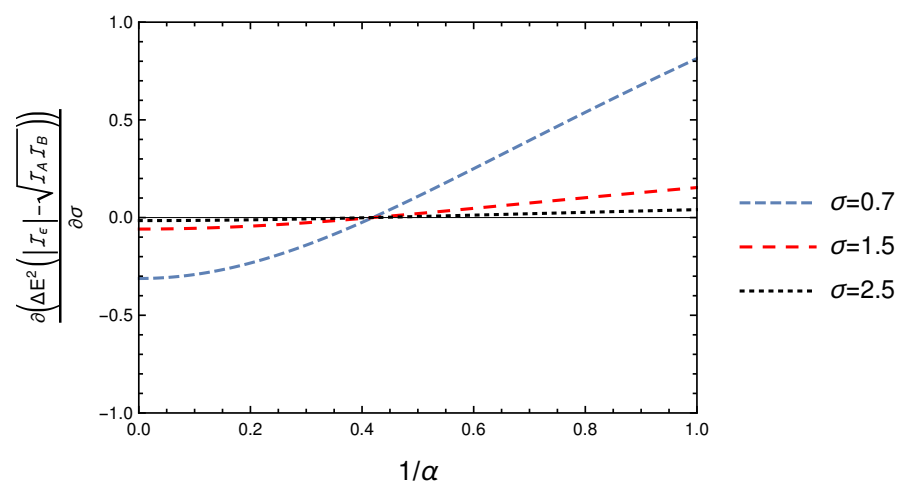

Figure 18. In $(1+3)$ dimensions the quantity $(\partial / \partial \sigma)\left(\Delta E^{2}\left(\left|\mathcal{I}_{\varepsilon}\right|-\sqrt{\mathcal{I}_{A} \mathcal{I}_{B}}\right)\right)$ is plotted for two anti-parallelly accelerating detectors with respect to the equal magnitude of proper accelerations $\alpha_{A}=\alpha_{B}$ for fixed inverse temperature of the thermal bath $\sigma$.

\section{Mutual information}

From eq. (2.12) and (2.13) it is observed that the mutual information corresponding to the two accelerated detectors interacting with background thermal field can be estimated by estimating the quantities $P_{j}$ and $P_{A B}$. From eq. (4.1) and (4.2) one can find out the expressions of $P_{j}$ in $(1+1)$ and $(1+3)$ dimensions corresponding to observers accelerated parallelly or anti-parallelly. Then here we only have to find out the expression of $P_{A B}$ to understand the nature of the mutual information for the considered detector pair. In particular we are going to estimate $\mathcal{I}_{A B}$ of (2.4) from which it is straightforward to estimate $P_{A B}$ using eq. (2.3). We shall first consider the prallelly and then anti-parallelly accelerated detectors to estimate these quantities.

\subsection{Parallel acceleration}

\subsection{1 (1+1) dimensions}

We consider the Wightman function of eq. (3.14) corresponding to parallelly accelerated detectors interacting with thermal fields, and consider a change of variables $\tilde{v}=\tau_{B}+\tau_{A}$ 
and $\tilde{u}=\tau_{B}-\tau_{A}$ to evaluate the integral $\mathcal{I}_{A B}$ as

$$
\begin{aligned}
\mathcal{I}_{A B}= & \int_{-\infty}^{\infty} d \tau_{B} \int_{-\infty}^{\infty} d \tau_{A} e^{i\left(\Delta E^{A} \tau_{A}-\Delta E^{B} \tau_{B}\right)} G_{W_{R}}^{\beta}\left(X_{B}, X_{A}\right) \\
= & \delta\left(\frac{\Delta E^{A}-\Delta E^{B}}{\sqrt{a_{A} a_{B}}}\right) \frac{\pi}{\Delta \widetilde{E} \sqrt{a_{A} a_{B}}} \frac{1}{\sqrt{\sinh \frac{\pi \Delta \widetilde{E}}{a_{B}} \sinh \frac{\pi \Delta \widetilde{E}}{a_{A}}}} \\
& {\left[\frac{e^{-\frac{\pi \Delta \widetilde{E}}{2}\left(\frac{1}{a_{B}}+\frac{1}{a_{A}}\right)}}{1-e^{-\beta \Delta \widetilde{E}}}+\frac{e^{\frac{\pi \Delta \widetilde{E}}{2}\left(\frac{1}{a_{B}}+\frac{1}{a_{A}}\right)}}{e^{\beta \Delta \widetilde{E}}-1}\right], }
\end{aligned}
$$

where $\Delta \widetilde{E}=\left(\Delta E^{B}+\Delta E^{A}\right) / 2$. It is to be noted that when $\Delta E^{B} \neq \Delta E^{A}$, due to the Dirac delta distribution $\delta\left(\left(\Delta E^{A}-\Delta E^{B}\right) / \sqrt{a_{A} a_{B}}\right)$ in front of the expression (5.1), the quantity $\mathcal{I}_{A B}$ (i.e., $P_{A B}$ ) vanishes. Then one can observe from eq. (2.13) that the quantities $P_{ \pm}$ become $P_{A}$ and $P_{B}$, which in turn leads to zero value of the mutual information from (2.12).

One has non vanishing mutual information only when $P_{A B} \neq 0$, i.e., when $\Delta E^{B}=$ $\Delta E^{A}$. We get $\Delta \widetilde{E}=\Delta E$ by considering $\Delta E^{B}=\Delta E^{A}=\Delta E$. In that case it is observed that there will be a multiplicative $\delta(0)$ term in the expression of $\mathcal{I}_{A B}$ similar to the case of $\mathcal{I}_{j}$ of (4.1). One can remove this $\delta(0)$ term attributed to considering a rate per unit proper time of $\mathcal{I}_{A B}$. On the other hand, from (2.3) it is observed that for the exact evaluation of $P_{A}, P_{B}$, and $P_{A B}$ it is imperative to know the expectation value $\left\langle E_{1}^{j}\left|m_{j}(0)\right| E_{0}^{j}\right\rangle$, which can be estimated for an explicit choice of the monopole operator $m_{j}(0)=\left|E_{1}^{j}\right\rangle\left\langle E_{0}^{j}|+| E_{0}^{j}\right\rangle\left\langle E_{1}^{j}\right|$. It is to be noted that in the expression of the concurrence from (2.9) there was a common multiplicative term $\left|\left\langle E_{1}^{B}\left|m_{B}(0)\right| E_{0}^{B}\right\rangle\right|\left|\left\langle E_{1}^{A}\left|m_{A}(0)\right| E_{0}^{A}\right\rangle\right|$, which we neglected concentrating only on the effect of the spacetime on detector response. However, for the case of the mutual information of (2.12) one cannot pull out a common multiplicative expectation of the monopole operator and we have to explicitly put their values for a numerical evaluation. In particular, for both $j=A$ and $j=B$ it is observed that $\left\langle E_{1}^{j}\left|m_{j}(0)\right| E_{0}^{j}\right\rangle=1$. Then using eq. (2.3), (2.4), (2.12), and (2.13) one can explicitly evaluate the mutual information in this case. In figure 19 we have plotted the rate of mutual information with respect to the temperature of the thermal field $T^{(f)}(\sim 1 / \sigma=1 /(\beta \Delta E))$, which shows that with increasing temperature the mutual information increases. From this figure it is also observed that with increasing acceleration of the first detector (signified by $\alpha_{A}$ ) the mutual information decreases.

\section{$5.1 .2(1+3)$ dimensions}

We consider the positive frequency Wightman function (3.16) for the estimation of the quantity $\mathcal{I}_{A B}$ in $(1+3)$ dimensions, which becomes

$$
\begin{aligned}
\mathcal{I}_{A B} & =\int_{-\infty}^{\infty} d \tau_{B} \int_{-\infty}^{\infty} d \tau_{A} e^{i\left(\Delta E^{A} \tau_{A}-\Delta E^{B} \tau_{B}\right)} G_{W_{R}^{3 D}}^{\beta}\left(X_{B}, X_{A}\right) \\
& =\delta\left(\frac{\Delta E^{A}-\Delta E^{B}}{\sqrt{a_{A} a_{B}}}\right) \frac{1}{\pi a_{A} a_{B}} \Upsilon\left(\Delta \widetilde{E}, a_{A}, a_{B}\right)\left[\frac{e^{-\frac{\pi \Delta \widetilde{E}}{2}\left(\frac{1}{a_{B}}+\frac{1}{a_{A}}\right)}}{1-e^{-\beta \Delta \widetilde{E}}}+\frac{e^{\frac{\pi \Delta \widetilde{E}}{2}\left(\frac{1}{a_{B}}+\frac{1}{a_{A}}\right)}}{e^{\beta \Delta \widetilde{E}}-1}\right] .
\end{aligned}
$$




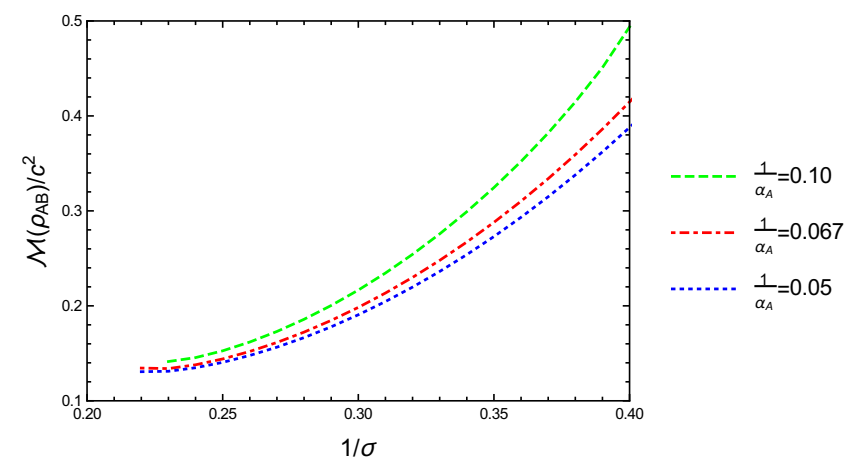

Figure 19. In $(1+1)$ dimensions the quantity $\mathcal{M}\left(\rho_{A B}\right) / c^{2}$ per unit proper time is plotted, which signifies the mutual information, for two parallelly accelerating detectors with respect to the temperature of the thermal field $T^{(f)}(\sim 1 / \sigma)$ for different fixed proper accelerations $\alpha_{A}$, where $\alpha_{B}=1$.

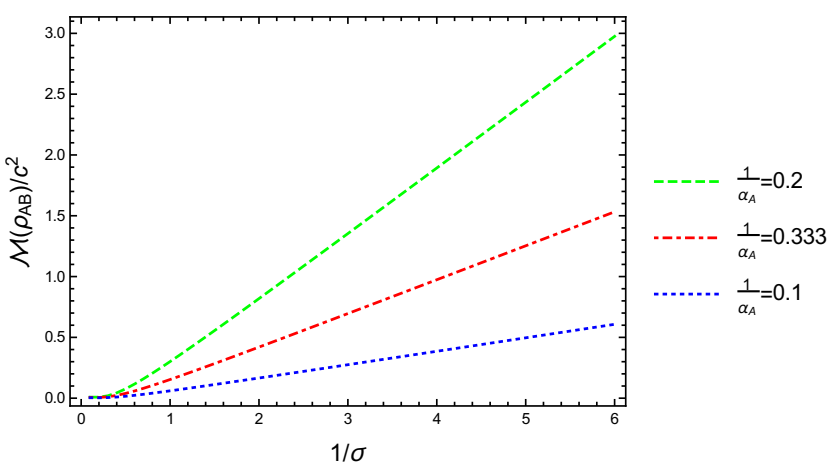

Figure 20. In $(1+3)$ dimensions the quantity $\mathcal{M}\left(\rho_{A B}\right) / c^{2}$ per unit proper time is plotted, which signifies the mutual information, for two parallelly accelerating detectors with respect to the temperature of the thermal field $T^{(f)}(\sim 1 / \sigma)$ for different fixed proper accelerations $\alpha_{A}$, where $\alpha_{B}=1$.

Here also $\Delta \widetilde{E}=\left(\Delta E^{B}+\Delta E^{A}\right) / 2$, and for $\Delta E^{B} \neq \Delta E^{A}$ Dirac delta distribution $\delta\left(\left(\Delta E^{A}-\Delta E^{B}\right) / \sqrt{a_{A} a_{B}}\right)$ in $(5.2)$ provides vanishing $\mathcal{I}_{A B}$ (or $P_{A B}$ ). This leads to vanishing mutual information.

The mutual information is non vanishing only when $\Delta E^{B}=\Delta E^{A}$. Here also considering $\left\langle E_{1}^{j}\left|m_{j}(0)\right| E_{0}^{j}\right\rangle=1$ we have estimated the mutual information using the eq. (2.3), (2.4), (2.12), and (2.13), and we plotted the rate of mutual information with respect to the temperature of the thermal field $T^{(f)}(\sim 1 / \sigma)$ in figure 20. From this figure we conclude that in $(1+3)$ dimensions also the mutual information increases with increasing temperature of the thermal field and decreasing acceleration of the first detector (signified by $\left.\alpha_{A}\right)$.

\subsection{Anti-parallel acceleration}

\subsection{1 (1+1) dimensions}

We consider the Wightman function from eq. (3.15) corresponding to two anti-parallelly accelerated observers in $(1+1)$ dimensional thermal bath, and a change of variables $\tilde{v}=$ 
$\tau_{B}+\tau_{A}$ and $\tilde{u}=\tau_{B}-\tau_{A}$ to evaluate the quantity $\mathcal{I}_{A B}$ from (2.4). One can express this integral $\mathcal{I}_{A B}$ as

$$
\begin{aligned}
\mathcal{I}_{A B}= & \int_{-\infty}^{\infty} d \tau_{B} \int_{-\infty}^{\infty} d \tau_{A} e^{i\left(\Delta E^{A} \tau_{A}-\Delta E^{B} \tau_{B}\right)} G_{W_{L R}}^{\beta}\left(X_{B}, X_{A}\right) \\
= & \delta\left(\frac{\Delta E^{A}+\Delta E^{B}}{\sqrt{a_{A} a_{B}}}\right) \frac{\pi}{\Delta \breve{E} \sqrt{a_{A} a_{B}}} \frac{1}{\sqrt{\sinh \frac{\pi \Delta \breve{E}}{a_{B}} \sinh \frac{\pi \Delta \breve{E}}{a_{A}}}} \\
& {\left[\frac{e^{-\frac{\pi \Delta \breve{E}}{2}\left(\frac{1}{a_{B}}-\frac{1}{a_{A}}\right)}}{1-e^{-\beta \Delta \breve{E}}}+\frac{e^{\frac{\pi \Delta \breve{E}}{2}\left(\frac{1}{a_{B}}-\frac{1}{a_{A}}\right)}}{e^{\beta \Delta \breve{E}}-1}\right] }
\end{aligned}
$$

where $\Delta \breve{E}=\left(\Delta E^{B}-\Delta E^{A}\right) / 2$. Then it is obvious that for $\Delta E^{A}>0$ and $\Delta E^{B}>0$ the Dirac delta distribution sitting in front of this expression $\delta\left(\left(\Delta E^{A}+\Delta E^{B}\right) / \sqrt{a_{A} a_{B}}\right)$ will provide a vanishing contribution. Thus $\mathcal{I}_{A B}$ vanishes and so vanishes $P_{A B}$. Then from eq. (2.13) one can estimate the quantities $P_{ \pm}$to be $P_{A}$ and $P_{B}$, which in turn leads to the expression of mutual information from $(2.12)$ to be vanishing upto $\mathcal{O}\left(c^{2}\right)$. This result persuades one to conclude that the mutual information corresponding to two anti-parallelly accelerated detectors in a thermal bath is zero in $(1+1)$ dimensions.

\section{$5.2 .2(1+3)$ dimensions}

We consider the positive frequency Wightman function (3.17) corresponding to antiparallelly accelerated observers for the estimation of the quantity $\mathcal{I}_{A B}$ in $(1+3)$ dimensions, which becomes

$$
\begin{aligned}
\mathcal{I}_{A B} & =\int_{-\infty}^{\infty} d \tau_{B} \int_{-\infty}^{\infty} d \tau_{A} e^{i\left(\Delta E^{A} \tau_{A}-\Delta E^{B} \tau_{B}\right)} G_{W_{L R}^{3 D}}^{\beta}\left(X_{B}, X_{A}\right) \\
& =\delta\left(\frac{\Delta E^{A}+\Delta E^{B}}{\sqrt{a_{A} a_{B}}}\right) \frac{1}{\pi a_{A} a_{B}} \Upsilon\left(\Delta \breve{E}, a_{A}, a_{B}\right)\left[\frac{e^{-\frac{\pi \Delta \breve{E}}{2}\left(\frac{1}{a_{B}}-\frac{1}{a_{A}}\right)}}{\left.1-e^{-\beta \Delta \breve{E}}+\frac{e^{\frac{\pi \Delta \breve{E}}{2}\left(\frac{1}{a_{B}}-\frac{1}{a_{A}}\right)}}{e^{\beta \Delta \breve{E}}-1}\right] .} .\right.
\end{aligned}
$$

Here also $\Delta \breve{E}=\left(\Delta E^{B}-\Delta E^{A}\right) / 2$ and similar to the $(1+1)$ dimensional case the Dirac delta distribution $\delta\left(\left(\Delta E^{A}+\Delta E^{B}\right) / \sqrt{a_{A} a_{B}}\right)$ sitting in front of this expression will provide a vanishing contribution. This leads to a vanishing $P_{A B}$ and in turn vanishing mutual information upto $\mathcal{O}\left(c^{2}\right)$ from (2.12). Then in $(1+3)$ dimensions also one can conclude that the mutual information corresponding to two anti-parallelly accelerated detectors in a thermal bath is zero.

\section{Discussion}

The possibility of constructing a plausible experimental setup in contact with a thermal bath is much higher, as, in nature, the background is thermal than a purely non-thermal field vacuum. Therefore it is much more relevant to understand realistic situations in our 
surroundings to study physical phenomena in the presence of a thermal bath or by considering the thermal fields. We have considered studying entanglement harvesting with two accelerated Unruh-DeWitt detectors interacting with a background thermal massless scalar field in this work. We have constructed the relevant Green's functions corresponding to accelerated observers in thermal bath considering the Rindler modes with the vacuum for the Unruh modes to avoid dealing with Wightman functions which are not time translational invariant. We used the prescription of [47] for constructing the Green's functions and followed the entanglement harvesting mechanism of articles $[38,40]$. It is observed that for zero temperature of the thermal bath, i.e., in the limit $\beta \rightarrow \infty$, in both $(1+1)$ and $(1+3)$ dimensions considering the equal magnitude of accelerations for the two observers but moving anti-parallelly, we always get the condition for entanglement harvesting to be satisfied. It ensures that entanglement extraction is possible for any finite non-zero acceleration in zero temperature background, which is in fact known from the earlier works of [4].

Furthermore, for non-zero temperature of the thermal bath with equal magnitude of acceleration of the anti-parallelly accelerated observers in both $(1+1)$ and $(1+3)$ dimensions we get identical conditions for entanglement harvesting (eq. (4.19)). The quantity $\mathcal{C}_{\mathcal{I}}$ signifying concurrence also shows similar behavior in $(1+1)$ and $(1+3)$ dimensions, which can be observed from figure 6,7 and 15, 16. An interesting fact we noticed from figure 7 and 16 is that with increasing temperature of the thermal bath (decreasing $\beta$ or $\sigma$ ), the range of acceleration, in which entanglement can be harvested, is decreasing, which is in agreement with the results of previous works [41, 42]. We observe that higher acceleration is needed to initiate entanglement harvesting with the higher temperature of the thermal bath. However, once for a certain temperature, entanglement harvesting starts with some initial acceleration in this system; it keeps on harvesting for all other higher accelerations. On the other hand, above a certain critical acceleration $a=a_{c}$ we see the amount of entanglement harvested, denoted by concurrence, to be increasing with increasing temperature of the thermal bath, showing a characteristic opposite compared to the region below $a=a_{c}$, which is like a phase transition.

We also observe in $(1+1)$ dimensions from eq. (4.12) and (4.16) that for $a_{A}=a_{B}=a$ and in the limit of $a \rightarrow 0$ the whole quantity $\mathcal{I}_{\varepsilon}$ without the multiplicative delta distribution vanishes, making the condition for entanglement extraction to break down. Then it is obvious that in the requirement of entanglement harvesting, an accelerated observer and a static observer in a thermal bath do not act in equal footing.

On the other hand, from earlier research works, it was known that the integral, representing the correlation between the two-detectors and responsible for mathematically realizing the entanglement harvesting, is related to the Wightman function $G_{W}\left(X_{B}^{\prime}, X_{A}\right)$ between two detector events. However, the recent investigations [38-40] suggest that this integral is related to the Feynman propagator $G_{F}\left(X_{B}^{\prime}, X_{A}\right)$. In particular, we observed that this additional contribution could be identified to be dependent on the Retarded Green's function. In our case we observed a finite contribution from the Retarded Green's function $G_{R}\left(X_{B}^{\prime}, X_{A}\right)$. In condition for entanglement extraction from eq. (4.17) and (4.23) we observed that the contribution from the Retarded Green's function can be identified through a quantity of $\sinh \left\{\pi \Delta E\left(1 / a_{B}-1 / a_{A}\right) / 2\right\}$. Then for all $a_{A} \neq a_{B}$ this quantity has a 
non-zero contribution. However, when $a_{A}=a_{B}$ the contributions from $G_{F}\left(X_{B}^{\prime}, X_{A}\right)$ and $G_{W}\left(X_{B}^{\prime}, X_{A}\right)$ are the same. Finally it is to be noticed that when $a_{A} \neq a_{B}$, by observing the plots of $\mathcal{C}_{\mathcal{I}}$ with respect to the acceleration of the first detector, it is possible to distinguish between the cases of $(1+1)$ and $(1+3)$ dimensions. Notably, in $(1+1)$ dimensions the curves of fixed $\sigma$ (figure 2) shows monotonic nature, while in $(1+3)$ dimensions (figure 10) this is not the case with various peaks and valleys. In $(1+3)$ dimensions this results in multiple transition points of accelerations $a_{A}$ between which the nature of concurrence with respect to the temperature of the thermal bath flips compared to the adjacent regions, also restricting the entanglement harvesting to discrete ranges of acceleration $a_{A}$ for certain fixed temperatures of the thermal bath.

An investigation of mutual information among the detectors has also been done here. We found that this vanishes for the anti-parallel situation, whereas it is non-vanishing for the parallel case. In the latter situation, mutual information increases with the increase in background field temperature while decreasing with the first detector's proper acceleration.

We want to mention here the nature of the curves in figure 7 and figure 16 which we did not discuss in the main text. In the equal acceleration case $a_{A}=a_{B}$ in both $(1+1)$ and $(1+3)$ dimensions, it is observed that after a specific critical acceleration, the entanglement extraction rate tends to decrease with increasing acceleration. The possible reason can be as follows. When the acceleration of the detector is substantial (i.e. $a_{A} \rightarrow \infty$ ), the detector moves very near to the null surface denoted by $X=-T$ and $X=T$ and also feels a very high temperature due to its acceleration (temperature is given by Unruh expression $a /(2 \pi))$. In this regime, the thermal bath due to acceleration becomes equally relevant along with the real thermal bath on the nature of entanglement harvesting. Since we already observed that temperature could reduce the entanglement between the detectors, both temperatures due to the Unruh effect and the thermal bath may play a role in the decreasing nature of concurrence. It is happening in a very high acceleration regime as there the Unruh temperature also becomes appreciable to affect entanglement harvesting. So in this regime, acceleration is showing its double standards - on one side, it is helping in entanglement, but on another side, it is also suppressing this phenomenon. In lower accelerations, the Unruh temperature is not so palpable to affect entanglement harvesting. Therefore there the acceleration plays only the role in helping entanglement. In this regard, we point out that this reason is only a suggestive one, and further investigation is needed to find any conclusive explanation.

Finally, we mention that in this paper, we deeply investigated the effect of background temperature on entanglement harvesting between two uniformly accelerated detectors. As we mentioned, this situation mimics a much more realistic situation, and hence the results have practical importance. As we mentioned above, the background temperature introduces several interesting noticeable features absent when the temperature is zero. Therefore we feel that the present study is significant in entanglement harvesting between the observers through their interaction with the background quantum fields and helps in the progress of the above subject. 


\section{Acknowledgments}

DB and SB would like to thank the Indian Institute of Technology Guwahati (IIT Guwahati) for supporting this work through Doctoral and Post-Doctoral Fellowships. The research of BRM is partially supported by a START-UP RESEARCH GRANT (No. $\mathrm{SG} / \mathrm{PHY} / \mathrm{P} / \mathrm{BRM} / 01$ ) from the Indian Institute of Technology Guwahati, India.

Open Access. This article is distributed under the terms of the Creative Commons Attribution License (CC-BY 4.0), which permits any use, distribution and reproduction in any medium, provided the original author(s) and source are credited.

\section{References}

[1] W. Tittel, J. Brendel, H. Zbinden and N. Gisin, Violation of Bell inequalities by photons more than 10 KM apart, Phys. Rev. Lett. 81 (1998) 3563 [quant-ph/9806043] [INSPIRE].

[2] D. Salart et al., Testing the speed of 'spooky action at a distance', Nature 454 (2008) 861.

[3] I. Fuentes-Schuller and R.B. Mann, Alice falls into a black hole: Entanglement in non-inertial frames, Phys. Rev. Lett. 95 (2005) 120404 [quant-ph/0410172] [INSPIRE].

[4] B. Reznik, Entanglement from the vacuum, Found. Phys. 33 (2003) 167 [quant-ph/0212044] [INSPIRE].

[5] S.-Y. Lin and B.L. Hu, Entanglement creation between two causally disconnected objects, Phys. Rev. D 81 (2010) 045019 [arXiv:0910.5858] [InSPIRE].

[6] J.L. Ball, I. Fuentes-Schuller and F.P. Schuller, Entanglement in an expanding spacetime, Phys. Lett. A 359 (2006) 550 [quant-ph/0506113] [INSPIRE].

[7] M. Cliche and A. Kempf, The relativistic quantum channel of communication through field quanta, Phys. Rev. A 81 (2010) 012330 [arXiv:0908.3144] [INSPIRE].

[8] E. Martin-Martinez and N.C. Menicucci, Cosmological quantum entanglement, Class. Quant. Grav. 29 (2012) 224003 [arXiv: 1204.4918] [INSPIRE].

[9] G. Salton, R.B. Mann and N.C. Menicucci, Acceleration-assisted entanglement harvesting and rangefinding, New J. Phys. 17 (2015) 035001 [arXiv: 1408.1395] [InSPIRE].

[10] E. Martin-Martinez, A.R.H. Smith and D.R. Terno, Spacetime structure and vacuum entanglement, Phys. Rev. D 93 (2016) 044001 [arXiv:1507.02688] [INSPIRE].

[11] H. Cai and Z. Ren, Transition processes of a static multilevel atom in the cosmic string spacetime with a conducting plane boundary, Sci. Rep. 8 (2018) 11802.

[12] G. Menezes, Entanglement dynamics in a Kerr spacetime, Phys. Rev. D 97 (2018) 085021 [arXiv: 1712.07151] [INSPIRE].

[13] G. Menezes, N.F. Svaiter and C.A.D. Zarro, Entanglement dynamics in random media, Phys. Rev. A 96 (2017) 062119 [arXiv: 1709.08702] [INSPIRE].

[14] W. Zhou and H. Yu, Resonance interatomic energy in a Schwarzschild spacetime, Phys. Rev. D 96 (2017) 045018 [INSPIRE].

[15] F. Benatti and R. Floreanini, Entanglement generation in uniformly accelerating atoms: Reexamination of the Unruh effect, Phys. Rev. A 70 (2004) 012112. 
[16] Y. Pan and B. Zhang, Influence of acceleration on multibody entangled quantum states, Phys. Rev. A 101 (2020) 062111 [arXiv: 2009.05179] [INSPIRE].

[17] A. Valentini, Non-local correlations in quantum electrodynamics, Phys. Lett. A 153 (1991) 321.

[18] B. Reznik, A. Retzker and J. Silman, Violating Bell's inequalities in the vacuum, Phys. Rev. A 71 (2005) 042104 [quant-ph/0310058] [INSPIRE].

[19] L.J. Henderson, R.A. Hennigar, R.B. Mann, A.R.H. Smith and J. Zhang, Harvesting Entanglement from the Black Hole Vacuum, Class. Quant. Grav. 35 (2018) 21LT02 [arXiv: 1712.10018] [INSPIRE].

[20] L.J. Henderson and N.C. Menicucci, Bandlimited Entanglement Harvesting, Phys. Rev. D 102 (2020) 125026 [arXiv:2005.05330] [INSPIRE].

[21] N. Stritzelberger, L.J. Henderson, V. Baccetti, N.C. Menicucci and A. Kempf, Entanglement harvesting with coherently delocalized matter, Phys. Rev. D 103 (2021) 016007 [arXiv: 2006.11291] [INSPIRE].

[22] M. Hotta, Quantum measurement information as a key to energy extraction from local vacuums, Phys. Rev. D 78 (2008) 045006 [arXiv: 0803.2272] [INSPIRE].

[23] M. Hotta, Quantum Energy Teleportation in Spin Chain Systems, J. Phys. Soc. Jpn. 78 (2009) 034001.

[24] M. Frey, K. Funo and M. Hotta, Strong local passivity in finite quantum systems, Phys. Rev. E 90 (2014) 012127.

[25] S. Hawking and W. Israel, General Relativity: an Einstein Centenary Survey, Cambridge University Press, Cambridge U.K. (2010).

[26] W.G. Unruh, Notes on black hole evaporation, Phys. Rev. D 14 (1976) 870 [INSPIRE].

[27] W.G. Unruh and R.M. Wald, What happens when an accelerating observer detects a Rindler particle, Phys. Rev. D 29 (1984) 1047 [InSPIRE].

[28] E. Martín-Martínez, E.G. Brown, W. Donnelly and A. Kempf, Sustainable entanglement production from a quantum field, Phys. Rev. A 88 (2013) 052310 [arXiv:1309.1090] [INSPIRE].

[29] K. Lorek, D. Pecak, E.G. Brown and A. Dragan, Extraction of genuine tripartite entanglement from the vacuum, Phys. Rev. A 90 (2014) 032316 [arXiv:1405.4449] [INSPIRE].

[30] G.L. Ver Steeg and N.C. Menicucci, Entangling power of an expanding universe, Phys. Rev. D 79 (2009) 044027 [arXiv:0711.3066] [INSPIRE].

[31] E.G. Brown, W. Donnelly, A. Kempf, R.B. Mann, E. Martin-Martinez and N.C. Menicucci, Quantum seismology, New J. Phys. 16 (2014) 105020 [arXiv:1407.0071] [InSPIRE].

[32] A. Pozas-Kerstjens and E. Martin-Martinez, Harvesting correlations from the quantum vacuum, Phys. Rev. D 92 (2015) 064042 [arXiv:1506.03081] [INSPIRE].

[33] A. Pozas-Kerstjens and E. Martin-Martinez, Entanglement harvesting from the electromagnetic vacuum with hydrogenlike atoms, Phys. Rev. D 94 (2016) 064074 [arXiv: 1605.07180] [INSPIRE].

[34] S. Kukita and Y. Nambu, Harvesting large scale entanglement in de Sitter space with multiple detectors, Entropy 19 (2017) 449 [arXiv:1708.01359] [INSPIRE]. 
[35] A. Sachs, R.B. Mann and E. Martin-Martinez, Entanglement harvesting and divergences in quadratic Unruh-DeWitt detector pairs, Phys. Rev. D 96 (2017) 085012 [arXiv:1704.08263] [INSPIRE].

[36] J. Trevison, K. Yamaguchi and M. Hotta, Spatially Overlapped Partners in Quantum Field Theory, J. Phys. A 52 (2019) 125402 [arXiv:1807.03467] [InSPIRE].

[37] T. Li, B. Zhang and L. You, Would quantum entanglement be increased by anti-Unruh effect?, Phys. Rev. D 97 (2018) 045005 [arXiv: 1802.07886] [InSPIRE].

[38] J.-I. Koga, G. Kimura and K. Maeda, Quantum teleportation in vacuum using only Unruh-DeWitt detectors, Phys. Rev. A 97 (2018) 062338 [arXiv: 1804.01183] [INSPIRE].

[39] K.K. Ng, R.B. Mann and E. Martín-Martínez, New techniques for entanglement harvesting in flat and curved spacetimes, Phys. Rev. D 97 (2018) 125011 [arXiv:1805.01096] [InSPIRE].

[40] J.-i. Koga, K. Maeda and G. Kimura, Entanglement extracted from vacuum into accelerated Unruh-DeWitt detectors and energy conservation, Phys. Rev. D 100 (2019) 065013 [arXiv: 1906.02843] [INSPIRE].

[41] E.G. Brown, Thermal amplification of field-correlation harvesting, Phys. Rev. A 88 (2013) 062336 [arXiv: 1309.1425] [INSPIRE].

[42] P. Simidzija and E. Martín-Martínez, Harvesting correlations from thermal and squeezed coherent states, Phys. Rev. D 98 (2018) 085007 [arXiv:1809.05547] [InSPIRE].

[43] S.S. Costa and G.E.A. Matsas, Background thermal contributions in testing the Unruh effect, Phys. Rev. D 52 (1995) 3466 [gr-qc/9412030] [INSPIRE].

[44] S. Kolekar and T. Padmanabhan, Quantum field theory in the Rindler-Rindler spacetime, Phys. Rev. D 89 (2014) 064055 [arXiv: 1309.4424] [INSPIRE].

[45] L. Hodgkinson, J. Louko and A.C. Ottewill, Static detectors and circular-geodesic detectors on the Schwarzschild black hole, Phys. Rev. D 89 (2014) 104002 [arXiv:1401.2667] [INSPIRE].

[46] C. Chowdhury, S. Das, S. Dalui and B.R. Majhi, How robust is the indistinguishability between quantum fluctuation seen from noninertial frame and real thermal bath, Phys. Rev. D 99 (2019) 045021 [arXiv: 1902.06900] [INSPIRE].

[47] S. Barman and B.R. Majhi, Radiative process of two entangled uniformly accelerated atoms in a thermal bath: a possible case of anti-Unruh event, JHEP 03 (2021) 245 [arXiv:2101.08186] [INSPIRE].

[48] A.P.C.M. Lima, G. Alencar and R.R. Landim, Asymptotic states of accelerated qubits in nonzero background temperature, Phys. Rev. D 101 (2020) 125008 [arXiv:2002.02020] [INSPIRE].

[49] J. Hu and H. Yu, Entanglement dynamics for uniformly accelerated two-level atoms, Phys. Rev. A 91 (2015) 012327 [arXiv: 1501.03321] [INSPIRE].

[50] A. Peres, Separability criterion for density matrices, Phys. Rev. Lett. 77 (1996) 1413 [quant-ph/9604005] [INSPIRE].

[51] M. Horodecki, P. Horodecki and R. Horodecki, On the necessary and sufficient conditions for separability of mixed quantum states, Phys. Lett. A 223 (1996) 1 [quant-ph/9605038] [INSPIRE]. 
[52] K. Zyczkowski, P. Horodecki, A. Sanpera and M. Lewenstein, On the volume of the set of mixed entangled states, Phys. Rev. A 58 (1998) 883 [quant-ph/9804024] [INSPIRE].

[53] G. Vidal and R.F. Werner, Computable measure of entanglement, Phys. Rev. A 65 (2002) 032314 [quant-ph/0102117] [INSPIRE].

[54] J. Eisert and M.B. Plenio, A Comparison of entanglement measures, J. Mod. Opt. 46 (1999) 145 [quant-ph/9807034] [INSPIRE].

[55] I. Devetak and A. Winter, Distillation of secret key and entanglement from quantum states, Proc. Roy. Soc. A 461 (2005) 207.

[56] C.H. Bennett, D.P. DiVincenzo, J.A. Smolin and W.K. Wootters, Mixed state entanglement and quantum error correction, Phys. Rev. A 54 (1996) 3824 [quant-ph/9604024] [INSPIRE].

[57] S. Hill and W.K. Wootters, Entanglement of a pair of quantum bits, Phys. Rev. Lett. 78 (1997) 5022 [quant-ph/9703041] [INSPIRE].

[58] W.K. Wootters, Entanglement of formation of an arbitrary state of two qubits, Phys. Rev. Lett. 80 (1998) 2245 [quant-ph/9709029] [INSPIRE].

[59] L.C.B. Crispino, A. Higuchi and G.E.A. Matsas, The Unruh effect and its applications, Rev. Mod. Phys. 80 (2008) 787 [arXiv:0710.5373] [InSPIRE].

[60] N.D. Birrell and P.C.W. Davies, Quantum fields in curved space, Cambridge Monographs on Mathematical Physics, Cambridge University Press, Cambridge U.K. (1984).

[61] S. Carroll, Spacetime and geometry. An introduction to general relativity, Addison-Wesley, Boston U.S.A. (2003).

[62] G. Compère, J. Long and M. Riegler, Invariance of Unruh and Hawking radiation under matter-induced supertranslations, JHEP 05 (2019) 053 [arXiv: 1903.01812] [INSPIRE].

[63] A. Higuchi, S. Iso, K. Ueda and K. Yamamoto, Entanglement of the Vacuum between Left, Right, Future, and Past: The Origin of Entanglement-Induced Quantum Radiation, Phys. Rev. D 96 (2017) 083531 [arXiv: 1709.05757] [INSPIRE]. 